\title{
D-Serine and D-Alanine Regulate Adaptive Foraging Behavior in Caenorhabditis elegans via the NMDA Receptor
}

\author{
Yasuaki Saitoh, Masumi Katane, Tetsuya Miyamoto, Masae Sekine, Kumiko Sakai-Kato, and ${ }^{\circledR}$ Hiroshi Homma \\ Laboratory of Biomolecular Science, Graduate School of Pharmaceutical Sciences, Kitasato University, Tokyo 108-8641, Japan
}

D-Serine (D-Ser) is a coagonist for NMDA-type glutamate receptors and is thus important for higher brain function. D-Ser is synthesized by serine racemase and degraded by D-amino acid oxidase. However, the significance of these enzymes and the relevant functions of D-amino acids remain unclear. Here, we show that in the nematode Caenorhabditis elegans, the serine racemase homolog SERR-1 and D-amino acid oxidase DAAO-1 control an adaptive foraging behavior. Similar to many organisms, C. elegans immediately initiates local search for food when transferred to a new environment. With prolonged food deprivation, the worms exhibit a long-range dispersal behavior as the adaptive foraging strategy. We found that serr-1 deletion mutants did not display this behavior, whereas daao-1 deletion mutants immediately engaged in long-range dispersal after food removal. A quantitative analysis of D-amino acids indicated that D-Ser and D-alanine (D-Ala) are both synthesized and suppressed during food deprivation. A behavioral pharmacological analysis showed that the long-range dispersal behavior requires NMDA receptor desensitization. Long-term pretreatment with D-Ala, as well as with an NMDA receptor agonist, expanded the area searched by wild-type worms immediately after food removal, whereas pretreatment with D-Ser did not. We propose that D-Ser and D-Ala are endogenous regulators that cooperatively induce the long-range dispersal behavior in $C$. elegans through actions on the NMDA receptor.

Key words: D-amino acid oxidase; DAAO-1; forward movement; NMDA receptor desensitization; serine racemase; SERR-1

Significance Statement

In mammals, D-serine (D-Ser) functions as an important neuromodulator of the NMDA-type glutamate receptor, which regulates higher brain functions. In Caenorhabditis elegans, previous studies failed to clearly define the physiological significance of D-Ser, D-alanine (D-Ala), and their metabolic enzymes. In this study, we found that these D-amino acids and their associated enzymes are active during food deprivation, leading to an adaptive foraging behavior. We also found that this behavior involved NMDA receptor desensitization.

\section{Introduction}

Free D-serine (D-Ser) is highly concentrated in the mammalian forebrain (Hashimoto et al., 1993), where it binds to the glycinebinding sites of NMDA-type glutamate receptors to potentiate

\footnotetext{
Received 0ct. 2, 2019; revised Aug. 10, 2020; accepted Aug. 12, 2020.

Author contributions: Y.S., M.K., and H.H. designed research; Y.S. performed research; Y.S., M.K., T.M., M.S., and H.H. analyzed data; Y.S., K.S.-K., and H.H. wrote the paper.

This work was supported by Japan Society for the Promotion of Science Grants-in-Aid for Scientific Research Grants JP18K14907 (to Y.S.) and JP18K06117 (to M.K.) and by a Kitasato University Research Grant for Young Researchers (Y.S.). We thank Natsu Obara, Chisa Sakurai, Chiaki Miyakawa, Chisato Nakamura, Minako Watanabe, Masaki Ogawa, Haruka Kubo, and Megumi Harada for their excellent technical assistance. We also thank Dr. Gary Silverman for vector pAV1997, Dr. Peter Askjaer for plasmid pBN41, and Dr. Taro Sakamoto and Dr. Hirotaka Imai for microinjection support. The cosmid T01H8 was kindly provided by the Wellcome Trust Sanger Institute. Some strains were kindly supplied by the National Bioresource Project (Tokyo Women's Medical University) and the Caenorhabditis Genetics Center (University of Minnesota), which is funded by the National Institutes of Health, National Center for Research Resources.

The authors declare no competing financial interests.

Correspondence should be addressed to Hiroshi Homma at hommah@pharm.kitasato-u.ac.jp.

https://doi.org/10.1523/JNEUROSCI.2358-19.2020

Copyright $\odot 2020$ the authors
}

glutamatergic neurotransmission (Wolosker, 2007; Nishikawa, 2011) and thus contributes to the regulation of higher brain function. Astrocyte-derived and neuron-derived D-Ser regulates NMDA receptor-dependent long-term potentiation and depression in hypothalamic and hippocampal excitatory synapses (Panatier et al., 2006; Henneberger et al., 2010; Rosenberg et al., 2013), and altered D-Ser levels are implicated in the pathophysiology of various neuropsychiatric disorders, including schizophrenia (Bendikov et al., 2007), Alzheimer's disease (Wu et al., 2004), and amyotrophic lateral sclerosis (Sasabe et al., 2007). Free D-alanine (D-Ala), like D-Ser, is an NMDA receptor coagonist (Kleckner and Dingledine, 1988). However, under physiological conditions, its low concentrations and unknown biosynthetic pathway cast doubt on whether it functionally contributes to NMDA receptor activity.

We previously demonstrated that D-Ser and D-Ala are present in the nematode Caenorhabditis elegans (Saitoh et al., 2012), which has a well-described nervous system (White et al., 1986). We also showed that D-Ala but not D-Ser is present in 
Escherichia coli strain OP50, which is used as a food source for $C$. elegans (Saitoh et al., 2012). Thus, D-Ala detected in C. elegans is derived, at least in part, from the diet, whereas D-Ser is biosynthesized. D-Ser is synthesized by Ser racemase (SerR; EC 5.1.1.16), the enzyme that converts L-Ser to D-Ser (Wolosker et al., 1999; Inoue et al., 2008), and degraded by D-amino acid oxidase (DAO; EC 1.4.3.3), which stereospecifically acts on neutral and basic D-amino acids, including D-Ala (Krebs, 1935; Miyoshi et al., 2009). The daao-1 gene in C. elegans encodes a functional DAO (Katane et al., 2010), and the daao-1(tm3673) deletion mutant strain has reduced self-brood sizes and hatching (Saitoh et al., 2012), indicating DAAO-1 contributes to C. elegans fertility. However, D-Ser and D-Ala are present at similar levels in adult wild-type and daao-1(tm3673) mutant animals. C. elegans harbors a SerR homolog, SERR-1, which exhibits lower SerR activity than the mammalian enzyme (Uda et al., 2016; Katane et al., 2016). Nevertheless, D-Ser is present at similar levels in adult wild-type and serr-1(tm1988) deletion mutant animals (Katane et al., 2016). Thus, the physiological relationship between D-Ser and its metabolic enzymes, including DAAO-1 and SERR-1 in C. elegans, and their significance to higher brain function and behavior remain to be fully elucidated.

In this paper, to clarify this issue, we first examined various behaviors of daao-1(tm3673) and serr-1(tm1988) mutants. We found that these mutants exhibit abnormal foraging behaviors during food deprivation. We then performed quantitative analyses of D-amino acids and behavioral pharmacological analyses in food-deprived C. elegans to determine how D-amino acids and their metabolic enzymes contribute to foraging behaviors. The results revealed that endogenous $\mathrm{D}$-Ser and $\mathrm{D}$-Ala, as well as DAAO-1 and SERR-1, control an adaptive foraging behavior in C. elegans by regulating NMDA receptor activity.

\section{Materials and Methods \\ C. elegans strains}

The Bristol strain N2 was used as the standard wild-type strain. The mutant and transgenic strains used in this study are listed in Table 1. These mutant and transgenic strains were backcrossed five times. Unless otherwise noted, all strains were grown at $20^{\circ} \mathrm{C}$ on nematode growth medium agar plates seeded with E. coli strain OP50 (Brenner, 1974).

\section{Assays for behavioral phenotypes}

Pharyngeal pumping

Pharyngeal pumping rates were measured as described previously (Raizen et al., 1995) with some modifications. Briefly, day 1 adult hermaphrodites grown at $25^{\circ} \mathrm{C}$ (the day after larval stage late L4) were individually placed on agar plates seeded with thick bacterial lawns. The pharynx of each worm was video recorded for $10 \mathrm{~s}$ under a M205 C stereomicroscope equipped with an MC170 HD camera (Leica). Pumping rates were measured by counting grinder movements using the slowmotion video replay.

\section{Defecation}

Worm defecation is conducted by three distinct motor steps: posterior body muscle contraction (pBoc), anterior body muscle contraction (aBoc), and expulsion muscle contraction (EMC; Thomas, 1990). To measure the defecation interval, day 1 adult hermaphrodites grown at $25^{\circ} \mathrm{C}$ were individually placed on agar plates seeded with thick bacterial lawns. For each worm, the period required for 10 consecutive defecation cycles was measured under the stereomicroscope. On the basis of these measurements, the time of a defecation interval (from one pBoc to the next $\mathrm{pBoc}$ ) was calculated. To measure the EMC rate, the numbers of the resultant EMCs in the 10 consecutive trials were measured for each worm.
Table 1. List of $C$. elegans strains

\begin{tabular}{ll}
\hline C. elegans strain & Source (identifier) \\
\hline Wild-type & Caenorhabditis Genetics left (N2) \\
daao-1(tm3673) & National Bioresource Project \\
serr-1(tm1988) & National Bioresource Project \\
$n m r-1(a k 4)$ & Caenorhabditis Genetics left (VM487) \\
$n m r-2(t m 3785)$ & National Bioresource Project \\
$n m r-1(a k 4) ; n m r-2(t m 3785)$ & This paper \\
daao-1(tm3673);nmr-1(ak4) & This paper \\
daao-1(tm3673);nmr-2(tm3785) & This paper \\
daao-1(tm3673);serr-1(tm1988) & This paper \\
daao-1(tm3673) Ex[daao-1::mCherry] & This paper \\
serr-1(tm1988) Ex[T01H8] & This paper \\
Ex[nrap-1::mCherry] & This paper \\
daao-1(tm3673) Ex[nrap-1:::mCherry] & This paper \\
akls3[Pnmr-1::GFP] & Caenorhabditis Genetics left (VM484) \\
daao-1(tm3673) akls3[Pnmr-1::GFP] & This paper \\
serr-1(tm1988) akls3[Pnmr-1::GFP] & This paper \\
\hline
\end{tabular}

Nose touch response

Nose touch assays were performed as described previously (Kaplan and Horvitz, 1993) with some modifications. Briefly, day 1 adult hermaphrodites grown at $25^{\circ} \mathrm{C}$ were individually placed on food-free agar plates. A fine hair was placed on the surface of the agar in worm's path, and its movements were observed under the stereomicroscope. The numbers of resultant backing responses in eight consecutive trials were measured for each worm.

\section{Anterior and posterior body touch responses}

Anterior and posterior body touch assays were performed as described previously (Chalfie and Sulston, 1981) with some modifications. Briefly, day 1 adult hermaphrodites grown at $25^{\circ} \mathrm{C}$ were individually placed on agar plates seeded with thin bacterial lawns. To evoke the anterior and posterior body touch responses, the worms were touched by stroking an eyelash across the body just behind the pharynx and just before the anus, respectively. Worms were observed under the stereomicroscope. The numbers of the resultant backward and forward movements in five consecutive trials were measured for each worm.

\section{Body bend}

Day 2 adult hermaphrodites grown at $20^{\circ} \mathrm{C}$ were individually placed on food-free agar plates. Body bends were counted for 2 min under the stereomicroscope, and this value was used to calculate the number of body bends per minute. A body bend was defined as maximum flexure of the body just behind the pharynx in the direction opposite to the previously scored bend.

\section{Food searching strategies}

Local search behavior. Well-fed day 2 adults grown at $20^{\circ} \mathrm{C}$ were placed individually on unseeded agar plates (observation plates). The forward and backward movement times for the first four movements were measured under the stereomicroscope at room temperature (20$22^{\circ} \mathrm{C}$ ), and the average time for each movement was calculated. The reversal frequencies were calculated as the numbers of each movement per minute (these were defined as the times of forward and backward movements and the reversal frequencies $0-5 \mathrm{~min}$ after food removal).

Long-range dispersal behavior assay. Well-fed day 2 adults grown at $20^{\circ} \mathrm{C}$ were placed individually on unseeded agar plates (transition plates) and cultured for $60 \mathrm{~min}$ before being transferred to observation plates for measurements of forward and backward movements as described above. The $10-\mathrm{cm}$-diameter observation and transition plates were prepared the day of or $2 \mathrm{~d}$ before the assays and dried at room temperature for $1 \mathrm{~h}$ just before the assays were performed. For pharmacological experiments, agar plates containing various concentrations of drugs were prepared with stock solutions of $700 \mathrm{~mm}$ NMDA ( $\mathrm{pH}$ 8.5, adjusted with $\mathrm{NaOH}$ ), $41.7 \mathrm{~mm}$ MK-801, $1 \mathrm{~m}$ D-Ser, $1 \mathrm{~m} \mathrm{~L}-S e r$, and $1 \mathrm{~m}$ D-Ala dissolved in sterile water. The short-term treatments were performed by culturing day 2 adults on seed drug plates for $2 \mathrm{~h}$. The long-term 
Table 2. List of plasmids used for generation of transgenic strains

\begin{tabular}{ll}
\hline Vector or recombinant DNA & Source (identifier) \\
\hline pAV1997 & Addgene (RRID: Addgene_37831) \\
pAV1997(42_132del) & This paper \\
mCherry::PTS1 CDNA & This paper (pYS1) \\
pAV1997(42_132del)-PTS1 & This paper (pYS2) \\
Pdaao-1 & This paper (pYS3) \\
Pdaao-1::mCherry::PTS1 & This paper (pYS4) \\
daao-1 & This paper (pYS5) \\
daao-1::mCherry::PTS1 & This paper (pYS6) \\
nrap-1 & This paper (pYS7) \\
Pnrap-1 & This paper (pYS8) \\
nrap-1::mCherry & This paper (pYS9) \\
serr-1 clone & Wellcome Trust Sanger Institute (T01H8) \\
Pmyo-2:::GFP & Addgene (pBN41, RRID: Addgene_86716) \\
\hline
\end{tabular}

Table 3. List of primer sequences used for construction of transgene plasmids

\begin{tabular}{lll}
\hline Primer pair & Forward primer & Reverse primer \\
\hline$\# 1$ & GTGGACTCTAGCATGGTGAGCAAGGGC & GATATCCTACAACTTCTTCGACTTGTA \\
& CAGCTCGTCCATGC \\
$\# 2$ & GCATGCGATCTTCCGTATCCGCC & GTCGACTTCTGAAAAATATAGA \\
$\# 3$ & GTCGACATGCCTAAAATTGCTGTAC & GTCGACCTTTTTCATTTCAGCAC \\
$\# 4$ & GCATGCTGGAACAGTGTTTGCCTCAT & CCCGGGTTCTCGTGATTACCTGCAAT \\
\hline
\end{tabular}

treatments were performed by culturing worms of various stages on seed drug plates to day 2 adults. For pharmacological experiments 60-65 min after food removal, the transition plates containing the same concentrations of drugs were used.

\section{Construction of transgene plasmids and generation of transgenic strains}

The provided or constructed plasmids and the primer sequences are listed in Tables 2, 3, respectively. The promoterless mCherry (a red fluorescent protein) vector, pAV1997 (Miedel et al., 2012), was revised by deletion of the XbaI-NheI region containing start and stop codons [pAV1997(42_132del)]. To generate pAV1997(42_132del)-encoding mCherry with a C-terminal peroxisomal targeting signal type 1 (PTS1) sequence, a full-length mCherry coding sequence was amplified by PCR using pAV1997 as the template and primer pair \#1. The forward primer of primer pair \#1 contains an additional Sall site at the $5^{\prime}$ end, and the reverse primer of primer pair \#1 contains an additional EcoRV site with the PTS1 sequence at the $5^{\prime}$ end. The PCR product was cloned into vector pTA2 (Toyobo, Osaka, Japan), and the construct was confirmed using sequencing (pYS1). Subsequently, the SalI-EcoRV fragment of pYS1 was subcloned into pAV1997(42_132del) (pYS2).

To generate the daao-1 promoter-DAAO-1-mCherry fusion construct (daao-1::mCherry), a 1987-bp genomic DNA fragment upstream of the daao-1 initiation codon was amplified by PCR using wild-type worm genomic DNA as the template and primer pair \#2. The forward and reverse primers of primer pair $\# 2$ contain additional SphI and SalI sites at their $5^{\prime}$ ends, respectively. The PCR product was cloned into pTA2, and the construct was confirmed by sequencing (pYS3). Subsequently, the SphI-SalI fragment of pYS3 was subcloned into pYS2, yielding the Pdaao-1::mCherry plasmid (pYS4). A full-length daao-1 genomic DNA fragment, lacking the PTS1 sequence, was amplified by PCR using wild-type worm genomic DNA as the template and primer pair \#3. The forward and reverse primers of primer pair \#3 contain additional SalI sites at their $5^{\prime}$ ends. The PCR product was cloned into pTA2, and the construct was confirmed using sequencing (pYS5). Subsequently, the SalI fragment of pYS5 was subcloned into pYS4, resulting in the daao1::mCherry plasmid (pYS6).

The nrap-1 promoter-NRAP-1-mCherry fusion construct (nrap-1:: $m$ Cherry) was generated as described previously (Lei et al., 2017) with some modifications. A full-length nrap-1 genomic DNA fragment, which lacks the intron 3 and four sequences and contains an additional
XmaI site at the $5^{\prime}$ end and NheI site at the $3^{\prime}$ end, was chemically synthesized (Genewiz) and cloned into pAV1997 (pYS7). A 4803-bp genomic DNA fragment upstream of the nrap-1 initiation codon was amplified by PCR using wild-type worm genomic DNA as the template and primer pair \#4. The forward and reverse primers of primer pair \#4 contain additional SphI and XmaI sites at their 5' ends, respectively. The PCR product was cloned into pTA2, and the construct was confirmed using sequencing (pYS8). Subsequently, the SphI-XmaI fragment of pYS8 was subcloned into pYS7, resulting in the nrap-1::mCherry plasmid (pYS9).

Germline transgene experiments were performed as described previously (Mello et al., 1991). Briefly, transgenic lines were generated by injecting $90 \mathrm{ng} / \mu \mathrm{l}$ pYS6, along with $10 \mathrm{ng} / \mu \mathrm{l}$ pBN41 (Morales-Martínez et al., 2015) as the transformation marker, into the gonads of daao-1(tm3673) mutant hermaphrodites (daao-1 (tm3673) Ex[daao-1::mCherry]) or $20 \mathrm{ng} / \mu \mathrm{l} \mathrm{T01H8,} \mathrm{along} \mathrm{with} 4 \mathrm{ng} /$ $\mu \mathrm{pBN} 41$ and $76 \mathrm{ng} / \mu \mathrm{l}$ pUC19 as the carrier DNA, into the gonads of serr-1(tm1988) mutant hermaphrodites (serr-1(tm1988) Ex[T01H8]) or $50 \mathrm{ng} / \mu \mathrm{l}$ pYS9, along with $4 \mathrm{ng} / \mu \mathrm{l} \mathrm{pBN} 41$ and $46 \mathrm{ng} / \mu \mathrm{l} \mathrm{pUC} 19$, into the gonads of wild-type hermaphrodites (Ex[nrap-1::mCherry]). Transgenic worms were identified by the expression of pharyngeal GFP from pBN41, and stable lines were isolated.

\section{Microscopic analysis}

Day 2 adult hermaphrodites, while on food and 1 or $60 \mathrm{~min}$ after food removal, were mounted on $2 \%$ agar pads with a drop of $10 \mathrm{~mm}$ sodium azide solution. Confocal images were acquired on an LSM710 microscope with ZEN software (Zeiss).

\section{Determination of amino acid contents}

Synchronized L1 larvae prepared using a standard alkaline bleach treatment (Lewis and Fleming, 1995) were placed on seeded agar plates and cultured at $20^{\circ} \mathrm{C}$ for $3 \mathrm{~d}$. The worms (corresponding to day 1 adults) were collected and either washed three times with cold water and stored at $-80^{\circ} \mathrm{C}$ or washed twice with M9 buffer and placed on $15-\mathrm{cm}$-diameter food-free agar plates. The excess liquid was removed with a soft paper wipe, and the worms were cultured at $20^{\circ} \mathrm{C}$ for 1,10 , or $60 \mathrm{~min}$. After removing worms that crawled up the plate wall, the cultured worms were collected, washed twice with cold water, and then stored at $-80^{\circ} \mathrm{C}$.

Whole-body extracts were prepared and D- and L-amino acids were quantitatively analyzed by HPLC as described previously (Katane et al., 2016) with some modifications. The samples were sonicated in $750 \mu \mathrm{l}$ of $100 \%(\mathrm{v} / \mathrm{v})$ acetonitrile using a Sonifier 250 analog cell disruptor (Branson), followed by incubation at $-80^{\circ} \mathrm{C}$ for $\geq 1 \mathrm{~d}$. The lysates were centrifuged twice at $20,000 \times g$ for $5 \mathrm{~min}$ at $4^{\circ} \mathrm{C}$ to remove precipitated proteins. The supernatants were then evaporated to dryness, and the residue was dissolved in $100 \mu \mathrm{l}$ of $400 \mathrm{~mm}$ borate buffer ( $\mathrm{pH} 9.0$ ). The solutions were cleared by centrifugation at $20,000 \times g$ for $5 \mathrm{~min}$ at $4^{\circ} \mathrm{C}$, and the supernatants were filtered through $0.45-\mu \mathrm{m}$ Millex-LH filters (Millipore) and stored at $-20^{\circ} \mathrm{C}$. A total of $30 \mu \mathrm{l}$ of sample diluted with $400 \mathrm{~mm}$ borate buffer ( $\mathrm{pH} 9.0$ ) was mixed with $10 \mu \mathrm{l}$ of water and $20 \mu \mathrm{l}$ of $o$-phthalaldehyde (OPA)/N-tert-butyloxycarbonyl-L-cysteine (Boc-LCys) reagent [prepared by mixing $10 \mathrm{mg}$ of OPA with $10 \mathrm{mg}$ of Boc-LCys in $1 \mathrm{ml}$ of $100 \%(\mathrm{v} / \mathrm{v})$ methanol] to generate fluorescent derivatives of the amino acids. After incubating at room temperature for $2 \mathrm{~min}$, $10 \mu \mathrm{l}$ of the mixture was injected into the Jasco chromatographic system comprising a model PU-2089 pump, a model FP-2025 fluorescence detector, and a model 807-IT integrator (Jasco Corp.). The amino acid derivatives were separated on an octadecylsilyl silica gel column (Mightysil RP-18GP, $150 \times 4.6 \mathrm{~mm}$ in internal diameter; Kanto Chemical Co) at a flow rate of $1 \mathrm{ml} / \mathrm{min}$, with gradients comprising solution A (50 mm sodium acetate buffer, $\mathrm{pH}$ 6.0) and solution B (100\% (v/v) acetonitrile] as follows: $0-40 \mathrm{~min}, 10 \%$ to $18 \%$ solution $\mathrm{B} ; 40-$ $50 \mathrm{~min}, 18-25 \%$ solution $\mathrm{B}$; $50-51 \mathrm{~min}, 25-50 \%$ solution B; and $51-$ $61 \mathrm{~min}, 50 \%$ solution B. Fluorescence was detected at an excitation wavelength of $344 \mathrm{~nm}$ and an emission wavelength of $443 \mathrm{~nm}$. The amounts of D- and L-amino acids were determined on the basis of the peak areas in the chromatograms. 
Table 4. List of primer sequences used for real-time PCR

\begin{tabular}{lll}
\hline Gene & Forward primer & Reverse primer \\
\hline tba-1 & ACCAACAAGCCGATGGAGAA & CAGTGGGATCTCATCAACA \\
rskn-1 & CTGCATTACGCTTTCCAAC & AGTGCCAATGTGAGTTCTGC \\
serr-1 & CTGGAAGTTTCAAAGCCAGAGG & GAATGGGCAATCATTCCTTTG \\
daao-1 & AGTCTATCCGACGCGTGGTC & TGTGGAGTCGTTCAACAGCG \\
\hline
\end{tabular}

\section{Quantification of mRNA expression}

Total RNA extraction and cDNA synthesis were performed as described previously (Ly et al., 2015) with some modifications. Briefly, three wildtype day 2 adult hermaphrodites grown at $20^{\circ} \mathrm{C}$ and cultured on foodfree agar plates at $20^{\circ} \mathrm{C}$ for 1,10 , or $60 \mathrm{~min}$ were placed in $5 \mu \mathrm{l}$ of lysis buffer containing $1 \mathrm{mg} / \mathrm{ml}$ proteinase $\mathrm{K}$ in a $0.2 \mathrm{ml}$ tube. The tube was incubated at $65^{\circ} \mathrm{C}$ for $10 \mathrm{~min}$ and then $85^{\circ} \mathrm{C}$ for $1 \mathrm{~min}$ to inactivate proteinase $\mathrm{K}$, and the lysate was used immediately for cDNA synthesis. cDNA synthesis was performed using Maxima $\mathrm{H}$ Minus cDNA synthesis master mix with dsDNase (Thermo Fisher Scientific) as described in the manufacturer's manual, and the cDNA was stored $-80^{\circ} \mathrm{C}$.

For quantitative real-time PCR, $2 \mu \mathrm{l}$ of the diluted cDNA was mixed with $10 \mu \mathrm{l}$ of SsoFast EvaGreen supermix (Bio-Rad), $1 \mu \mathrm{l}$ of $10 \mu \mathrm{M}$ forward primer, $1 \mu \mathrm{l}$ of $10 \mu \mathrm{M}$ reverse primer, and $6 \mu \mathrm{l}$ of RNase-free water. The mixture was subjected to PCR amplification using the CFX96 Touch real-time PCR detection system (Bio-Rad). The primer sequences are listed in Table 4 . The cycling conditions were as follows: initial denaturation at $95^{\circ} \mathrm{C}$ for $30 \mathrm{~s}$, followed by 40 cycles of denaturation at $95^{\circ} \mathrm{C}$ for $5 \mathrm{~s}$ and annealing and extension at $60^{\circ} \mathrm{C}$ for $5 \mathrm{~s}$. Raw threshold cycle $\left(C_{T}\right)$ values were determined using CFX Manager software (Bio-Rad) and used to calculate relative expression levels by the calibration curve method. The relative expression levels were normalized to $\alpha$-tubulin (tba-1) as the reference gene and compared with the means from the worms cultured with food.

\section{Experimental design and statistical analysis}

To evaluate the statistical significance of differences between two means, data were analyzed by Student's unpaired $t$ tests. When the variance differed between the two groups, the Welch's unpaired $t$ test was used. To evaluate the statistical significance of differences between the control mean and two or more means or among three or more means, data were analyzed by Dunnett's tests or by Tukey-Kramer tests, respectively. When the variance differed among the groups, the Games-Howell test was used. Statistical tests were conducted using JASP version 0.12.2. software (JASP Team 2020, University of Amsterdam).

Feeding, defecation, touch response, and body bend assays were designed to identify changes associated with genotype. For pharyngeal pumping, statistical difference between wild-type and daao-1(tm3673) worms was analyzed by Welch's unpaired $t$ test ( $n=14$ worms). For defecation interval, EMC/pBoc, nose touch, anterior body touch, and posterior body touch, Student's unpaired $t$ tests were used instead $(n=10,10$, 9,15 , and 15 worms, respectively). For body bend, statistical differences between wild-type and daao-1(tm3673) or serr-1(tm1988) worms were analyzed by Dunnett's test ( $n=6$ worms).

Foraging behavior assays were designed to identify changes associated with genotype, food conditions, and drug treatments. In Figure $1 C-E$, wild-type and daao-1(tm3673) worms $0-5$ and 60-65 min after food removal were examined ( $n=13$ worms). For forward movement time, statistical differences between each condition in each strain and versus wild-type under the same conditions were analyzed by Welch's unpaired $t$ tests. For backward movement time and reversal frequency, Student's unpaired $t$ tests were used instead. In Figure 1G-I, wild-type, daao-1(tm3673), and transgenic daao-1(tm3673) expressing the daao-1:: $m$ Cherry transgene $0-5$ and 60-65 min after food removal were examined ( $n=10$ worms). In Figure $1 L-N$, wild-type, serr-1(tm1988), and transgenic serr-1(tm1988) expressing the serr-1 transgene 0-5 and 60$65 \mathrm{~min}$ after food removal were examined ( $n=14$ worms). For forward movement time, statistical differences between each condition in each strain and among strains under the same conditions were analyzed by Welch's unpaired $t$ tests and Games-Howell tests, respectively. For backward movement time and reversal frequency, Student's unpaired $t$ tests and Tukey-Kramer tests, respectively, were used instead.

Quantitative analyses of D- and L-amino acids were designed to identify changes associated with genotype and food conditions (Figure $2 \mathrm{~A}-$ $D)$. The numbers of whole-body worm extracts analyzed were as follows: for wild-type and daao-1(tm3673), $n=7,4$, 4, and 6 trials for on food, 1 , 10, and $60 \mathrm{~min}$ after food removal, respectively; for $\operatorname{serr}-1$ (tm1988), $n=$ $5,4,5$, and four trials for on food, 1,10 , and $60 \mathrm{~min}$ after food removal, respectively. Statistical differences among conditions in each strain and versus wild-type under the same conditions were analyzed by TukeyKramer tests or by Dunnett's tests, respectively.

Quantitative analyses of mRNA expression of rskn-1, serr-1, and daao- 1 were designed to identify changes associated with genotype and food conditions. In Figure $1 K$, Student's unpaired t test was employed for statistical difference between well-fed wild-type and F2 serr-1 (tm1988) worms ( $n=4$ trials). In Figure 2E,F, Tukey-Kramer tests were employed for statistical differences among conditions; wild-type worms on food or 1, 10, or $60 \mathrm{~min}$ after food removal ( $n=4$ trials).

The forward movement times of day 2 adults deprived of food for 0 5 min were analyzed unless otherwise noted. In Figure $3 A$, GamesHowell test was employed for statistical differences among strains; wild-type, $n m r-1(a k 4), n m r-2(t m 3785)$, and $n m r-1(a k 4) ; n m r-2(t m 3785)$ worms $(n=13$ worm). In Figure $3 B$, Student's unpaired $t$ tests were employed for statistical differences versus untreated group in each strain; wild-type, $n m r-1(a k 4)$, and $n m r-2(t m 3785)$ worms untreated or treated with $900 \mu \mathrm{M}$ MK-801 short term ( $n=11$ worms). In Figure 3C, GamesHowell test was employed for statistical differences among groups; wildtype worms untreated or treated with $900 \mu \mathrm{M}$ MK-801 and/or $5 \mathrm{~mm}$ NMDA short term ( $n=11$ worms). In Figure $3 D$, Dunnett's test was employed for statistical differences versus untreated group; wild-type worms untreated or treated with $5 \mathrm{~mm}$ NMDA long-term ( $n=9$ worms). In Figure $3 E$, Student's unpaired $t$ tests were employed for statistical differences versus untreated group in each strain; $n m r-1$ (ak4) and $n m r-2$ (tm3785) worms untreated or treated with $5 \mathrm{~mm}$ NMDA long-term ( $n=9$ worms). In Figure 3F, Games-Howell test was employed for statistical differences among groups; wild-type worms untreated or treated with $100 \mu \mathrm{M}$ MK-801 and/or 5 mM NMDA long-term ( $n=12$ worms). In Figure 3G, Tukey-Kramer test was employed for statistical differences among groups; day 3 adult wild-type worms untreated or treated with 5 mm NMDA long-term ( $n=10$ worms). In Figure 3I, Games-Howell test was employed for statistical differences among groups; wild-type and transgenic wild-type expressing the nrap-1::mCherry transgene untreated or treated with $5 \mathrm{~mm}$ NMDA long-term ( $n=10$ worms). In Figure $4 A$, Games-Howell test was employed for statistical differences among groups; wild-type worms untreated or treated with $900 \mu \mathrm{M}$ MK-801 and/ or $10 \mathrm{~mm} \mathrm{D}$-Ser short term ( $n=9$ worms). In Figure $4 B$, Student's unpaired $t$ test was employed for statistical difference versus untreated group; wild-type worms untreated or treated with $10 \mathrm{~mm}$ D-Ser longterm ( $n=12$ worms). In Figure $4 C$, Games-Howell test was employed for statistical differences among groups; wild-type worms untreated or treated with $900 \mu \mathrm{M}$ MK-801 and/or $10 \mathrm{mM}$ D-Ala short term $(n=12$ worms). In Figure $4 D$, Games-Howell test was employed for statistical differences versus untreated group; wild-type worms untreated or treated with $10 \mathrm{~mm}$ D-Ala long-term ( $n=8-9$ worms). In Figure $4 E$, Student's unpaired $t$ tests were employed for statistical differences versus untreated group in each strain; $n m r-1(a k 4)$ and $n m r-2(t m 3785)$ worms untreated or treated with $10 \mathrm{~mm}$ D-Ala long-term ( $n=12-14$ worms). In Figure $4 F$, Games-Howell test was employed for statistical differences among groups; wild-type worms untreated or treated with $100 \mu \mathrm{M}$ MK801 and/or $10 \mathrm{~mm}$ D-Ala long-term ( $n=12$ worms). In Figure $4 G$, Tukey-Kramer test was employed for statistical differences among groups; day 3 adult wild-type worms untreated or treated with $10 \mathrm{~mm} \mathrm{D-}$ Ala long-term ( $n=10$ worms). In Figure $4 H$, Games-Howell test was employed for statistical differences among groups; wild-type and transgenic wild-type expressing the nrap-1::mCherry transgene untreated or treated with $10 \mathrm{~mm}$ D-Ala long-term ( $n=10$ worms). In Figure $5 A$, Student's unpaired $t$ test was employed for statistical difference versus untreated group; daao-1(tm3673) worms untreated or treated with 900 $\mu \mathrm{M}$ MK-801 short term ( $n=7$ worms). In Figure $5 B$, Games-Howell test 
A

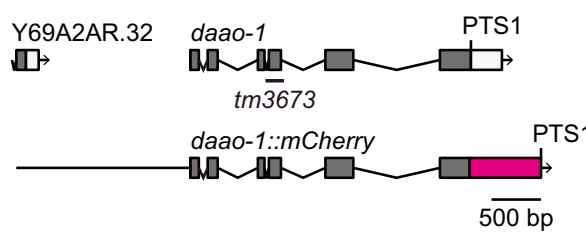

F

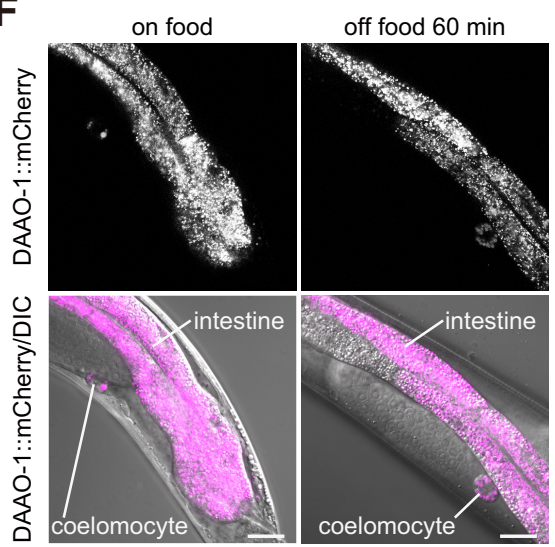

J

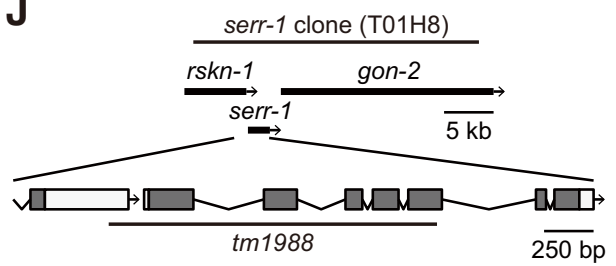

$K_{\text {, }}$

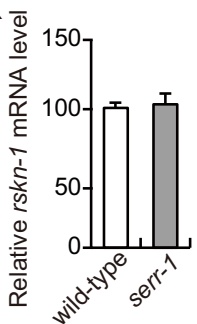

B

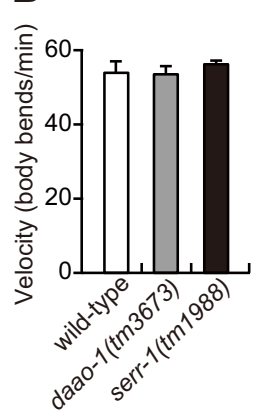

G
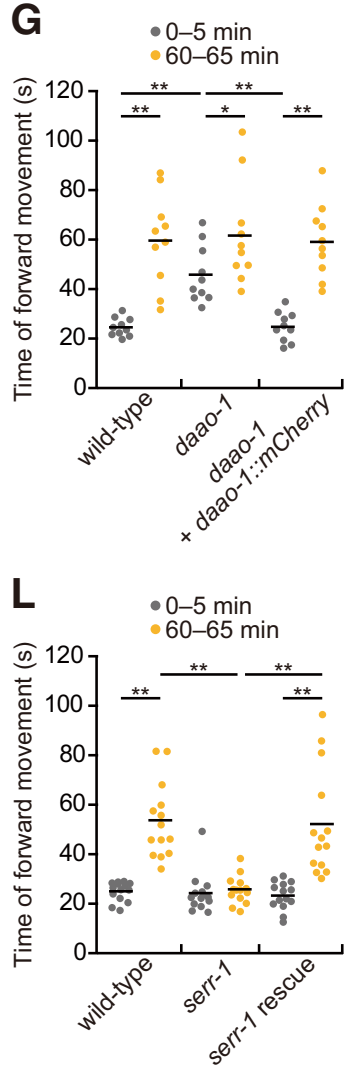

C

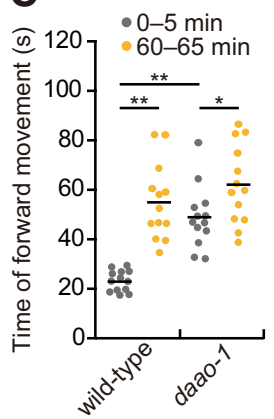

D

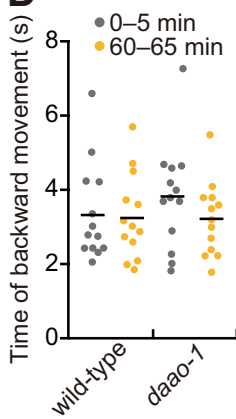

E
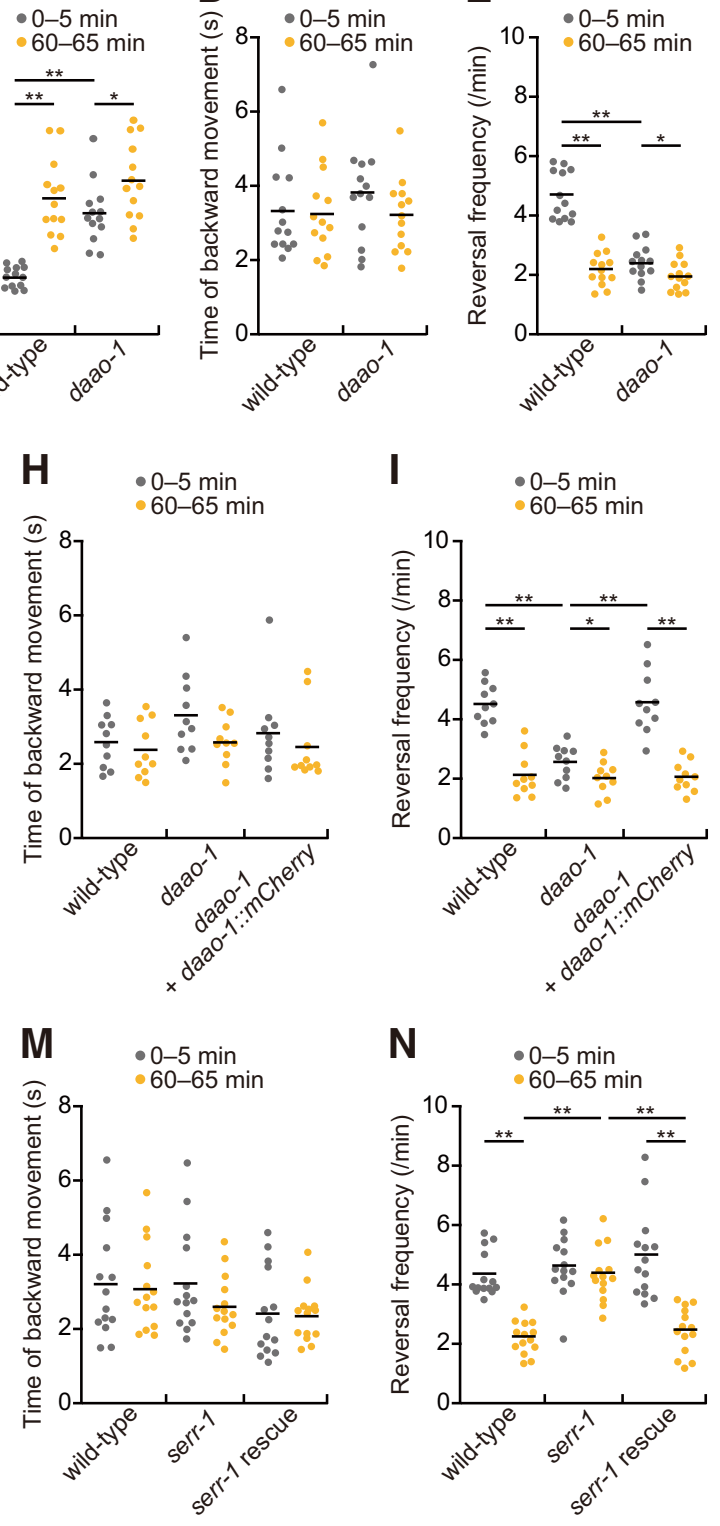

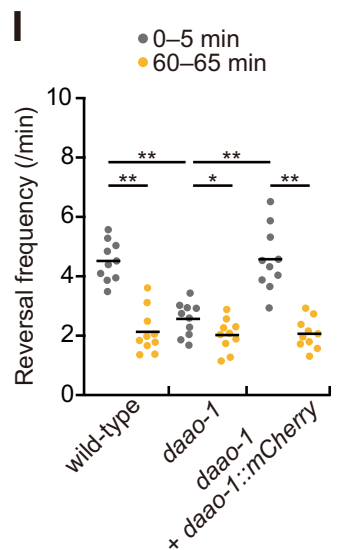

N

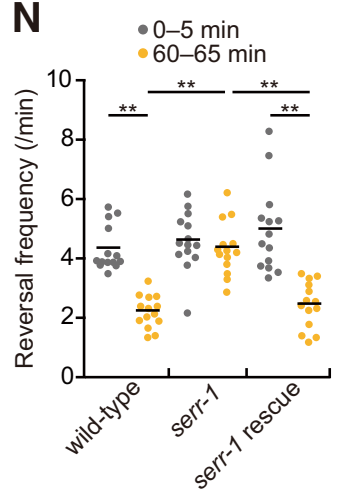

Figure 1. Foraging behavior of wild-type, daao-1 (tm 3673$)$, and serr-1(tm 1988) worms during food deprivation. A, Schematic representation of genomic structure of daao-1 and the transgene construct used in this study. Open, gray, and magenta boxes indicate the UTR, coding exon, and mCherry CDNA, respectively. The positions of the PTS1 and tm3673 deletion are indicated. In the daao-1::mCherry transgene construct, mCherry with a C-terminal PTS1 N-terminally fused with full-length DAA0-1 is expressed under the control of the daao-1 promoter. $\boldsymbol{B}$, Velocities of wild-type, daao-1(tm3673), and serr-1(tm1988) worms. Bar graphs represent means \pm SEM. Forward $(\boldsymbol{C}$ and backward $(\boldsymbol{D})$ movement times, and reversal frequencies $(\boldsymbol{E})$ of wild-type and daao-1 (tm3673) worms $0-5$ and 60-65 min after food removal. Dots represent single worms; bars are means. $\boldsymbol{F}$, Localization pattern of DAA0-1 in adult hermaphrodites. Confocal images and overlay of confocal and differential interference contrast (DIC) images of transgenic daao-1(tm3673) carrying the daao-1::mCherry transgene on the HT115(DE3) diet and 60 min after food removal. Scale bars: $20 \mu \mathrm{m}$. Forward $(\boldsymbol{G})$ and backward $(\boldsymbol{H})$ movement times, and reversal frequencies $(\boldsymbol{I})$ of wild-type, daa0-1(tm3673), and transgenic daao-1/(tm3673) expressing the daao-1:: $m$ Cherry transgene on the HT115(DE3) diet 0-5 and 60-65 min after food removal. Dots represent single worms; bars are means. J, Schematic representation of genomic structure of serr-1. Open and gray boxes indicate the UTR and coding exon, respectively. The positions of the $t m 1988$ deletion and T01H8 coding are indicated. $\boldsymbol{K}$, Relative $r$ skn- 1 mRNA expression in well-fed wild-type and serr-1(tm1988) worms. Bar graphs represent means \pm SEM. Forward $(\boldsymbol{L})$ and backward $(\boldsymbol{M})$ movement times, and reversal frequencies $(\boldsymbol{N})$ of wild-type, serr-1(tm1988), and transgenic serr-1(tm 1988) expressing the serr-1 transgene $0-5$ and 60-65 min after food removal. Dots represent single worms; bars are means; ${ }^{*} p<0.05$ and ${ }^{* *} p<0.01$ indicate significant differences. For details, see Materials and Methods, Experimental design and statistical analysis.

was employed for statistical differences among strains; wild-type, daao-1 (tm3673), nmr-1(ak4), daao-1(tm3673);nmr-1(ak4), nmr-2(tm3785), and daao-1(tm3673);nmr-2(tm3785) worms ( $n=12-15$ worms). In Figure $5 C$, Dunnett's test was employed for statistical differences versus untreated group; daao-1(tm3673) worms untreated or treated with $5 \mathrm{~mm}$ NMDA, $10 \mathrm{~mm}$ D-Ser, or $10 \mathrm{~mm}$ D-Ala long-term ( $n=11-13$ worms). In Figure 5D, Games-Howell test was employed for statistical differences among groups; wild-type and daao-1(tm3673) worms untreated or treated with $100 \mu \mathrm{M}$ MK-801 long-term ( $n=12$ worms). In Figure $5 E$,
Games-Howell test was employed for statistical differences among groups; wild-type, daao-1(tm3673), and transgenic wild-type and daao-1 (tm3673) expressing the nrap-1::mCherry transgene ( $n=8$ worms). In Figure 5F, Dunnett's test was employed for statistical differences versus untreated group; serr-1(tm1988) worms untreated or treated with $5 \mathrm{~mm}$ NMDA, 10 mM D-Ser, or $10 \mathrm{~mm}$ D-Ala long-term ( $n=12-15$ worms). In Figure 5G, Games-Howell test was employed for statistical differences among strains; wild-type, daao-1(tm3673), serr-1(tm1988), and daao-1 (tm3673);serr-1(tm1988) worms ( $n=11$ worms). Games-Howell test 
also was employed for statistical differences versus untreated daao-1(tm3673);serr-1(tm1988) worms; daao-1(tm3673);serr-1(tm1988) worms untreated or treated with $10 \mathrm{~mm}$ D-Ser, D-Ala, or L-Ser long-term ( $n=11$ worms). In Figure $6 A$, wild-type, $n m r-1(a k 4)$, $n m r-2(t m 3785), n m r-1(a k 4) ; n m r-2(t m 3785)$, and transgenic wild-type expressing the nrap-1::mCherry transgene $0-5$ and $60-65 \mathrm{~min}$ after food removal were examined ( $n=9-15$ worms). Statistical differences between each condition in each strain and versus wildtype for strains 60-65 min after food removal were analyzed by Welch's unpaired $t$ tests or by GamesHowell test, respectively. In Figure 6B, wild-type and $n m r-1(a k 4) ; n m r-2$ (tm3785) worms untreated or treated with $100 \mu \mathrm{M}$ MK-801 long-term $0-5$ or 60 65 min after food removal were examined $(n=9-13$ worms). Statistical differences among groups in wildtype and between untreated and treated $n m r-1(a k 4)$; $n m r-2(t m 3785)$ were analyzed by Games-Howell test or by Student's unpaired $t$ test, respectively. In Figure $6 C$, Games-Howell tests were employed for statistical differences versus untreated group in each strain; wild-type and serr-1(tm1988) worms untreated or treated with $10 \mathrm{~mm}$ D-Ser, D-Ala, or L-Ser long-term 60-65 min after food removal ( $n=7-13$ worms).

Confocal microscopic analyses of DAAO-1 protein localization and $n m r$-1-expressing neurons were designed to identify changes associated with genotype, food conditions, and drug treatments. The typical results were presented in Figures $1 F, 3 H, 5 H$. In Figure $1 F$, transgenic daao-1(tm3673) worms expressing the daao-1::mCherry transgene on food and $60 \mathrm{~min}$ after food removal were examined ( $n=10$ worms). In Figure $3 H$, transgenic wild-type worms expressing the Pnmr-1::GFP transgene untreated or treated with 5 mM NMDA or $10 \mathrm{~mm}$ D-Ala long-term $1 \mathrm{~min}$ after food removal were examined ( $n=10$ worms). In Figure $5 H$, transgenic wild-type, daao-1(tm3673), and serr-1(tm1988) expressing the Pnmr-1::GFP transgene on food or $60 \mathrm{~min}$ after food removal were examined ( $n=10$ worms).

\section{Results}

Daao-1 and serr-1 mutants exhibit abnormal foraging behaviors during food deprivation

To investigate the physiological significance of D-amino acids in foraging strategies, we first examined the various behaviors of daao-1(tm3673) mutants, in which the coding region of daao-1 is deleted (Fig. 1A). daao-1(tm3673) mutants displayed normal feeding (pharyngeal pumping, Welch's unpaired $t$ test, $t_{(15.76)}=0.68, p=0.505$; Table 5), defecation (Student's unpaired $t$ test: defecation interval, $t_{(18)}=1.66, p=0.114$; EMC/ pBoc, $t_{(18)}=0, \quad p=1.000$; Table 5), and touch responses (Student's unpaired $t$ test: nose, $t_{(16)}=0.17, p=0.864$; anterior body, $t_{(28)}=1.67, p=0.105$; posterior body, $t_{(28)}=0, p=1.000$; Table 5) and coordinately moved at a normal velocity (Dunnett's test, $t=0.13, p=0.988$; Fig. $1 B$ ). Similar to many organisms, $C$. elegans exhibits local search behavior, with short-term forward movement and frequent spontaneous backing (reversal), immediately after it is transferred to an unseeded agar plate (Wakabayashi et al., 2004; Hills et al., 2004; Gray et al., 2005). The behavior changes toward long-range dispersal behavior, where the frequency of reversal movements decreases and the frequency of long-term forward movements increases, when food is no longer encountered. Wild-type worms deprived of food for $0-5$ and $60-65 \mathrm{~min}$ spent 20 and $60 \mathrm{~s}$, respectively,
B $\square$ wild-type $\square$ serr-1 daao-1
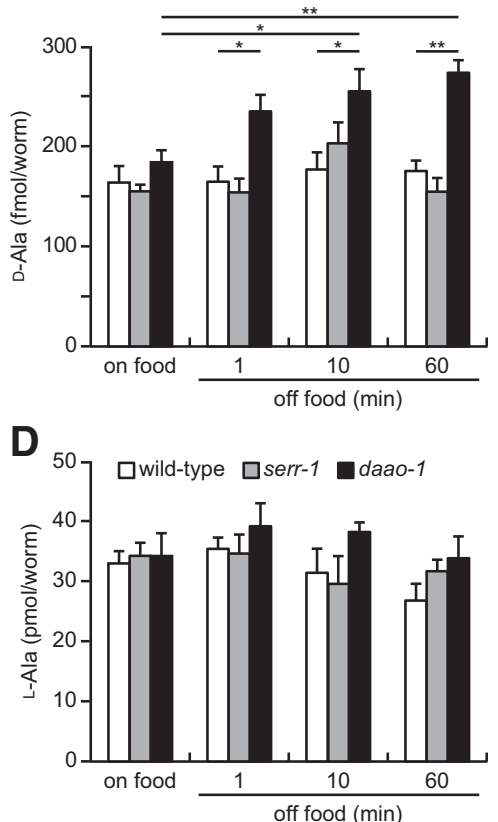
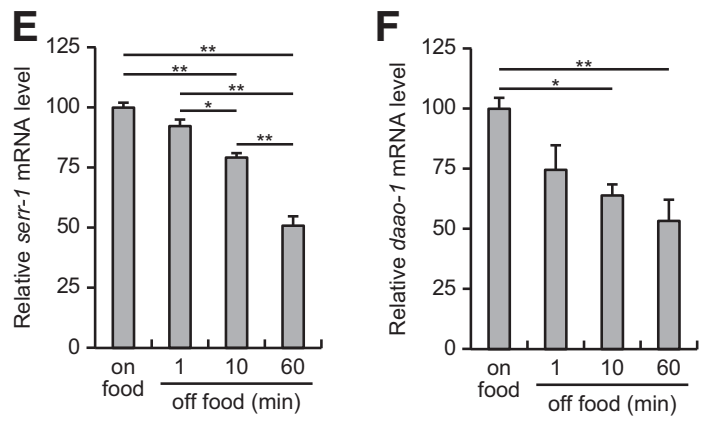

Figure 2. Amino acid levels and relative mRNA expression of daao-1 and serr-1. Contents of D-Ser $(\boldsymbol{A})$, D-Ala $(\boldsymbol{B})$, removal. Bar graphs represent means $\pm \mathrm{SEM} ;{ }^{*} p<0.05$ and ${ }^{* *} p<0.01$ indicate significant differences. For details, see Materials and Methods, Experimental design and statistical analysis.

moving forward (Welch's unpaired $t$ test, $t_{(13.80)}=7.25, p<$ 0.001; Fig. $1 C$ ) and $\sim 3$ s moving backward under each condition (Student's unpaired $t$ test, $t_{(24)}=0.16, p=0.876$; Fig. $1 D$ ), resulting in a reduction in the reversal frequency at $60-65 \mathrm{~min}$ after food removal (Student's unpaired $t$ test, $t_{(24)}=9.01, p<0.001$; Fig. $1 E)$. However, the forward movement time and reversal frequency after $0-5 \mathrm{~min}$ of deprivation were significantly longer and lower, respectively, in daao-1(tm3673) mutants than in wildtype worms [Welch's unpaired $t$ test, $t_{(14.69)}=7.11, p<0.001$ (Fig. 1C); Student's unpaired $t$ test, $t_{(24)}=8.38, p<0.001$ (Fig. $1 E)$ ] but were not different after 60-65 min [Welch's unpaired $t$ test, $t_{(23.98)}=1.17, p=0.255$ (Fig. $1 C$ ); Student's unpaired $t$ test, $t_{(24)}=1.20, p=0.241$ (Fig. $1 E$ )]. Under each condition, the backward movement times were similar between daao-1(tm3673) mutants and wild-type worms (Student's unpaired $t$ test: 0 5 min, $t_{(24)}=0.90, p=0.375 ; 60-65$ min, $t_{(24)}=0.09, p=0.928$; Fig. $1 D)$. To confirm that these phenotypes are because of the daao-1 mutation, we generated transgenic daao-1(tm3673) mutants carrying the daao-1::mCherry transgene, in which mCherry with a C-terminal PTS1 N-terminally fused with DAAO-1 under the control of the daao-1 promoter (Fig. 1A). The transgenic worms 
A

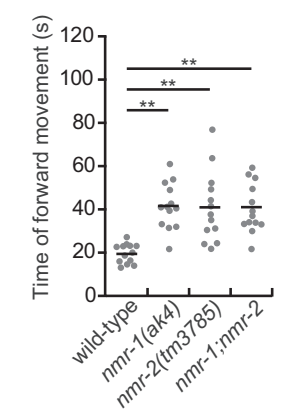

E

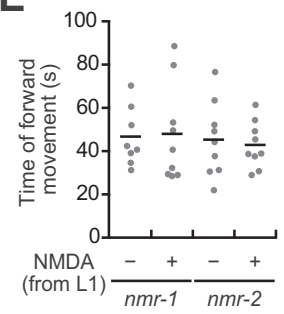

B

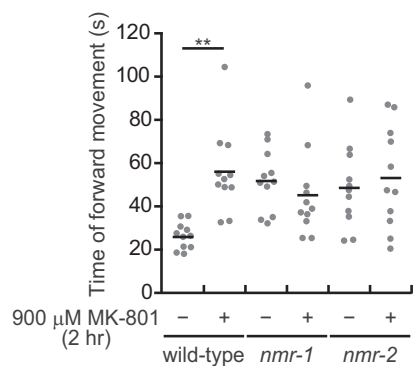

F

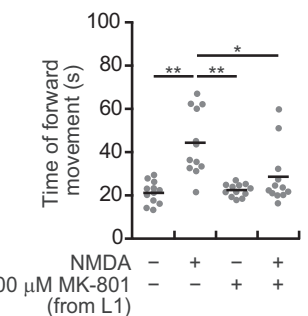

C

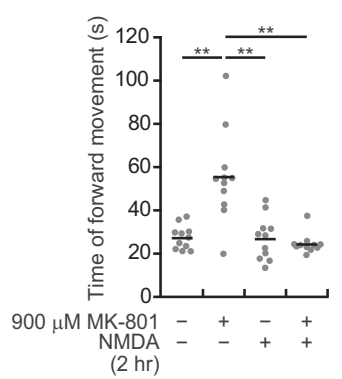

D

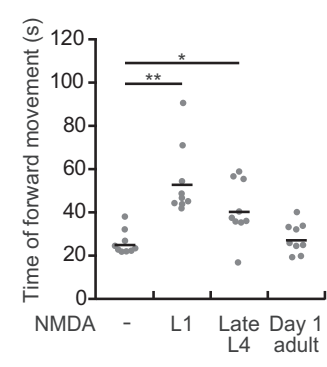

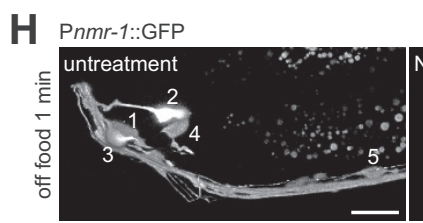

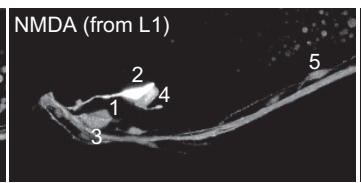

G

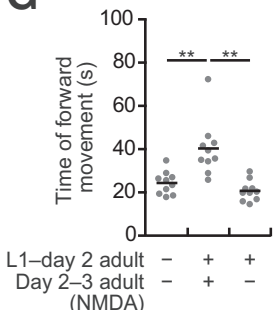

I

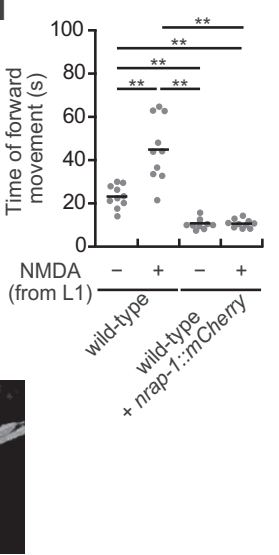

Figure 3. Effects of NMDA receptor agonist and antagonist on the time of forward movement immediately after food removal. $A-G, I$, Forward movement times $0-5$ min after food removal. Dots represent single worms; bars are means; ${ }^{*} p<0.05$ and ${ }^{* *} p<0.01$ indicate significant differences. $A$, Times of forward movement of $n m r-1(a k 4), n m r-2(t m 3785)$, and $n m r-1($ ak4),nmr-2(tm3785) worms. $B$, Effects of short-term treatments with high-dose MK-801 on the time of forward movement of wild-type, $n m r-1(a k 4)$, and $n m r-2$ (tm3785) worms. Day 2 adults were cultured for $2 \mathrm{~h}$ on seed agar plates containing $900 \mu \mathrm{m}$ MK-801. C, Effects of short-term treatments with NMDA and high-dose MK-801 on the times of forward movement of wild-type worms. Day 2 adults were cultured for $2 \mathrm{~h}$ on seed agar plates containing $5 \mathrm{~mm} \mathrm{NMDA}$ and/or $900 \mu \mathrm{m}$ MK-801. D, Effects of long-term treatments with NMDA on the times of forward movement of wild-type worms. The worms of L1 larvae, late L4 larvae, or day 1 adults were cultured to day 2 adults on seed agar plates containing $5 \mathrm{~mm} \mathrm{NMDA}$. $\boldsymbol{E}$, Effects of long-term treatments with NMDA on the times of forward movement of $n m r-1(a k 4)$ and $n m r-$ 2(tm3785) worms. L1 larvae were cultured to day 2 adults on seed agar plates containing $5 \mathrm{~mm} \mathrm{NMDA.} \boldsymbol{F}$, Effects of long-term treatments with NMDA and low-dose MK-801 on the times of forward movement of wild-type worms. L1 larvae were cultured to day 2 adults on seed agar plates containing 5 mM NMDA and/or $100 \mu \mathrm{m} \mathrm{MK-801.} \mathrm{G,} \mathrm{Times} \mathrm{of} \mathrm{forward} \mathrm{movements} \mathrm{of} \mathrm{day} 3$ adults. Wild-type L1 larvae were cultured with $5 \mathrm{~mm} \mathrm{NMDA}$ to day 2 adults and then cultured on seed drug-free agar plates for $1 \mathrm{~d}$. $\boldsymbol{H}$, Confocal stack images of transgenic wild-type worms expressing the Pnmr-1::GFP transgene 1 min after food removal. At least one of the four pairs of interneurons (1, AVA; 2, AVD; 3, AVE; 4, RIM) and an interneuron (5, AVG) in the left-side head region are shown. L1 larvae were cultured to day 2 adults on seed agar plates containing $5 \mathrm{~mm} \mathrm{NMDA}$ or $10 \mathrm{~mm} \mathrm{D-Ala}$. Scale bar: $20 \mu \mathrm{m}$. I, Effects of long-term treatments with NMDA on the times of forward movement of transgenic wildtype worms expressing the nrap-1::mCherry transgene. L1 larvae were cultured to day 2 adults on seed agar plates containing $5 \mathrm{~mm} \mathrm{NMDA.} \mathrm{For} \mathrm{details,} \mathrm{see} \mathrm{Materials} \mathrm{and} \mathrm{Methods,} \mathrm{Experimental} \mathrm{design}$ and statistical analysis.

Table 5. Behavioral analysis of daao-1 mutants

\begin{tabular}{lll}
\hline & \multicolumn{2}{l}{ Value (mean \pm SEM) } \\
Phenotype & Wild type & daao-1(tm3673) mutant \\
\hline Pharyngeal pumping (no. of pumps/10 s) & $47.5 \pm 0.8$ & $45.8 \pm 2.4^{a}$ \\
Defecation interval (s) & $42.5 \pm 0.9$ & $45.2 \pm 1.3^{b}$ \\
EMC/pBoc (\%) & $99.0 \pm 1.0$ & $99.0 \pm 1.0^{b}$ \\
Nose touch (\% response) & $84.7 \pm 5.4$ & $83.3 \pm 5.9^{b}$ \\
Anterior body touch (\% response) & $92.0 \pm 3.3$ & $84.0 \pm 3.5^{b}$ \\
Posterior body touch (\% response) & $84.0 \pm 4.0$ & $84.0 \pm 5.2^{b}$ \\
\hline
\end{tabular}

${ }^{a}$ Not significantly different from wild-type ( $p \geq 0.05$ by Welch's unpaired $t$ test).

${ }^{b}$ Not significantly different from wild-type ( $p \geq 0.05$ by the Student's unpaired $t$ test).

appeared debilitated on the OP50 diet but recovered through an unidentified mechanism on another standard E. coli strain HT115(DE3) diet. Under this culture condition, the localization of DAAO-1::mCherry on food and $60 \mathrm{~min}$ after food removal was observed in punctate structures, presumably peroxisomes, of intestinal cells and in the vesicles of coelomocytes (Fig. 1F). Although the foraging behavior of wild-type and daao-1 (tm3673) mutants on the HT115(DE3) diet was similar to that on the OP50 diet (Fig. $1 C-E, G-I$ ), the increased forward movement time and decreased reversal frequency of daao-1(tm3673) mutants after $0-5 \mathrm{~min}$ of deprivation were rescued by the expression of the daao-1::mCherry transgene [daao-1(tm3673) vs transgenic daao-1(tm3673): Games-Howell test, $t_{(13.52)}=5.07, p<$ 0.001 (Fig. 1G); Tukey-Kramer test, $t=5.58, p<0.001$ (Fig. 1I)]. Thus, daao-1(tm3673) mutants exhibit long-range dispersal behavior to search for food immediately after food removal.

We also examined foraging behaviors in serr-1(tm1988) mutants, in which both the coding and promoter regions of serr1 , as well a part of the $3^{\prime}$ untranslated region (UTR) of the upstream $r s k n-1$ gene, encoding a putative ribosomal protein S6 kinase, are deleted (Fig. 1J). However, a PCR analysis revealed rskn-1 mRNA expression did not differ between serr-1(tm1988) mutants and wild-type worms (Student's unpaired $t$ test, $t_{(6)}=0.29, p=0.779$; Fig. $\left.1 K\right)$; thus, these worms were considered specific serr-1 null mutants, which also moved at normal velocity (Dunnett's test, $t=0.70, p=0.708$; Fig. $1 B$ ). Although the foraging behavior of serr-1(tm1988) mutants after 0-5 min of food deprivation was similar to that of wild-type worms (Fig. $1 L-N)$, the serr-1(tm1988) mutants did not increase the time spent moving forward or decrease the reversal frequency after $60-65 \mathrm{~min}$ of food deprivation [0-5 vs 60-65 min in serr-1(tm1988): Welch's unpaired $t$ test, $t_{(23.35)}=0.59, p=0.563$ (Fig. $1 L$ ); Student's unpaired $t$ test, $t_{(26)}=0.77, p=0.450$ (Fig. 1N)]. The altered 
movement time and reversal frequency were rescued in transgenic serr-1(tm 1988) mutants that expressed a genomic serr-1 clone, cosmid T01H8 [serr-1(tm1988) versus transgenic serr-1 ( $t m 1988)$ : Games-Howell test, $t_{(14.78)}=4.42, p<0.001$ (Fig. $1 L$ ); Tukey-Kramer test, $t=6.56, p<0.001$ (Fig. $1 N$ )]. These results suggest that SERR-1 is essential for the long-range dispersal behavior after prolonged food deprivation.

\section{D-Ser and D-Ala in C. elegans are metabolized by SERR-1 and DAAO-1 in response to food deprivation}

We next performed HPLC to measure D-Ser and D-Ala levels in wild-type, daao-1(tm3673), and serr-1(tm1988) worms during food deprivation. In wild-type worms, D-Ser levels increased significantly $10 \mathrm{~min}$ after food removal and remained at a similar level $60 \mathrm{~min}$ after food removal (vs on food by Tukey-Kramer test: $1 \mathrm{~min}$ off food, $t=0.61, p=0.926 ; 10 \mathrm{~min}$ off food, $t=3.30$, $p=0.020$; $60 \mathrm{~min}$ off food, $t=4.22, p=0.003$; Fig. $2 A$ ). By contrast, no increase in D-Ser levels was observed in serr-1(tm1988) mutants (vs on food by Tukey-Kramer test: $1 \mathrm{~min}$ off food, $t=0.64, p=0.919 ; 10 \mathrm{~min}$ off food, $t=0.07, p=1.000$; $60 \mathrm{~min}$ off food, $t=0.80, p=0.856$; Fig. $2 A$ ), suggesting that SERR-1 is required for the synthesis of $\mathrm{D}$-Ser. However, D-Ser contents increased significantly within $1 \mathrm{~min}$ after food removal and remained high for up to $60 \mathrm{~min}$ after food removal in daao-1 (tm3673) mutants (vs on food by Tukey-Kramer test: $1 \mathrm{~min}$ off food, $t=4.14, p=0.003 ; 10 \mathrm{~min}$ off food, $t=4.18, p=0.003$; $60 \mathrm{~min}$ off food, $t=7.16, p<0.001$; Fig. $2 A$ ); the levels at $60 \mathrm{~min}$ were significantly higher than those in wild-type worms (Dunnett's test, $t=3.02, p=0.018$; Fig. $2 A$ ). D-Ala levels did not change markedly during food deprivation in wild-type (vs on food by TukeyKramer test: $1 \mathrm{~min}$ off food, $t=0.04, p=1.000 ; 10 \mathrm{~min}$ off food, $t=0.59, p=0.934 ; 60 \mathrm{~min}$ off food, $t=0.59, p=0.933$; Fig. $2 B$ ) or serr-1(tm1988) worms (vs on food by Tukey-Kramer test: $1 \mathrm{~min}$ off food, $t=0.05, p=1.000$; $10 \mathrm{~min}$ off food, $t=2.39, p=0.126 ; 60 \mathrm{~min}$ off food, $t=0.01, p=1.000$; Fig. $2 B$ ) but were increased remarkably $1 \mathrm{~min}$ after food removal in daao-1(tm3673) mutants and remained elevated for $60 \mathrm{~min}$ (vs on food by Tukey-Kramer test: $1 \mathrm{~min}$ off food, $t=2.38, p=0.119 ; 10 \mathrm{~min}$ off food, $t=3.32, p=0.019 ; 60 \mathrm{~min}$ off food, $t=4.72, p=0.001$; Fig. $2 B$ ). No marked changes in L-Ser (Fig. 2C) or L-Ala (Fig. 2D) levels were observed. These results suggest that both $\mathrm{D}$-Ser and D-Ala are synthesized in wild-type worms within $1 \mathrm{~min}$ after food removal and undergo degradation by DAAO-1 during food deprivation.

We next examined the relationship between altered D-amino acid metabolism and serr-1 and daao- 1 transcription. Specifically, we asked whether serr-1 expression increases and daao-1 expression decreases with the synthesis of D-Ser and D-Ala in wild-type worms. Unexpectedly, serr-1 expression decreased within $10 \mathrm{~min}$ after food removal, and decreased further after $60 \mathrm{~min}$ of food deprivation (vs on food by Tukey-Kramer test: $1 \mathrm{~min}$ off food, $t=1.97, p=0.251 ; 10 \mathrm{~min}$ off food, $t=5.33, p<0.001 ; 60 \mathrm{~min}$ off food, $t=12.61, p<0.001$; Fig. $2 E$ ). As predicted, daao- 1 decreased significantly during food deprivation (vs on food by TukeyKramer test: $1 \mathrm{~min}$ off food, $t=2.40, p=0.129 ; 10 \mathrm{~min}$ off food, $t=3.41, p=0.023 ; 60 \mathrm{~min}$ off food, $t=4.41, p=0.004$; Fig. $2 F$ ).

\section{NMDA receptor activity influences forward movement immediately after food removal}

D-Ser is a coagonist of the NR1 subunit that promotes calcium influx and postsynaptic membrane depolarization when L-glutamate binds to the NR2 subunit of the heteromeric complex in mammalian NMDA receptors (Traynelis et al., 2010). In the $C$. elegans nervous system, the homologs NMR-1 and NMR-2 colocalize exclusively in the command interneurons of the neural circuit that regulates locomotion (Chalfie et al., 1985; Brockie et al., 2001a). The glycine-binding site in NMR-1 is conserved (Kuryatov et al., 1994), and the C. elegans NMDA receptor is presumed to bind D-Ser and D-Ala. Previous studies showed that $n m r-1(a k 4)$ and $n m r-2(a k 7)$ deletion mutants spend more time moving forward but show normal velocities and similar backward movement times in comparison to wild-type animals (Brockie et al., 2001b; Lei et al., 2017). To investigate the role of NMDA receptor activity in $C$. elegans foraging behavior, we examined the movements of $n m r-1$ and $n m r-2$ deletion mutants immediately after food removal. Consistent with previous studies (Brockie et al., 2001b; Lei et al., 2017), nmr-1(ak4) mutants, as well as $n m r-2(t m 3785)$ mutants, exhibited significantly longer times of forward movements than wild-type worms (GamesHowell test: $n m r-1(a k 4), t_{(16.17)}=6.78, p<0.001 ; n m r-2(t m 3785)$, $t_{(13.83)}=4.53, p=0.002$; Fig. $\left.3 A\right)$. The time of forward movement of $n m r-1(a k 4) ; n m r-2(t m 3785)$ double mutants was similar to those of $n m r-1$ (ak4) and $n m r-2(t m 3785)$ single mutants (Games-Howell test: $t_{(15.69)}=6.10, p<0.001$ vs wild-type; $t_{(23.90)}=0.25, p=0.994$ vs $n m r-1(a k 4) ; t_{(21.45)}=0.10, p=1.000$ vs $n m r-2(t m 3785)$; Fig. $3 A$ ), suggesting that the NMDA receptor in C. elegans is also a heteromeric complex composed of NMR-1 and NMR-2 subunits and that the activity of these receptors regulates forward movement immediately after food removal. All worms tested in Figure $3 \mathrm{~A}$ moved coordinately and exhibited no obvious change in the time spent moving backward (data not shown).

Pharmacological activation of AVA command interneurons, which regulate backward movement, elicits a slow NMR-1-dependent and NMR-2-dependent outward current in patch-clamp recordings that is blocked when the noncompetitive antagonist MK-801 is applied (Brockie et al., 2001b; Kano et al., 2008). Thus, we assessed forward movement in well-fed day 2 adult worms incubated for $2 \mathrm{~h}$ on plates containing $900 \mu \mathrm{M}$ MK- 801 or $5 \mathrm{~mm}$ NMDA. We found that short-term treatment with the relatively high dose of MK-801 significantly increased the time wildtype worms spend moving forward but did not further increase that of $n m r-1(a k 4)$ or $n m r-2(t m 3785)$ mutants (Student's unpaired $t$ test: wild-type, $t_{(20)}=4.79, p<0.001 ; n m r-1(a k 4), t_{(20)}=0.87$, $p=0.395 ; n m r-2(t m 3785), t_{(20)}=0.50, p=0.624$; Fig. $\left.3 B\right)$. However, short-term treatment with NMDA did not affect the time of forward movement of wild-type worms but abolished the effect of high-dose MK-801 when coapplied (Games-Howell test: $t_{(15.61)}=$ $0.13, p=0.999$ between the untreated and NMDA-treated group; $t_{(10.93)}=4.73, p=0.003$ between the MK-801-treated and cotreated group; Fig. 3C). All worms tested in Figure $3 B, C$ moved coordinately and exhibited no obvious change in the backward movement time (data not shown). These results appear that agonism and antagonism of NMDA receptors elicit effects at the behavioral level.

We next examined the effects of longer-term treatment with NMDA and MK-801 on the time spent moving forward. For this, worms at various growth phases were incubated on seed plates containing $5 \mathrm{~mm}$ NMDA or $100 \mu \mathrm{M}$ MK-801 until they were at the day 2 adult stage. The time of forward movement of wild-type worms was not affected by NMDA treatment from the day 1 adult stage but it increased significantly when treatment was initiated at larval stages L1 and late L4 (Dunnett's test: day 1 adult, $t=0.40, p=0.958$; L1, $t=5.18, p<0.001$; late L4, $t=2.85$, $p=0.020$; Fig. $3 D$ ). However, $n m r-1(a k 4)$ and $n m r-2(t m 3785)$ single mutants treated as L1 larvae did not show increased forward movement times (Student's unpaired $t$ test: $n m r-1(a k 4)$, $t_{(15)}=0.14, p=0.894 ; n m r-2(t m 3785), t_{(16)}=0.36, p=0.726$; Fig. $3 E)$. The NMDA-induced increased time of forward movement 
A

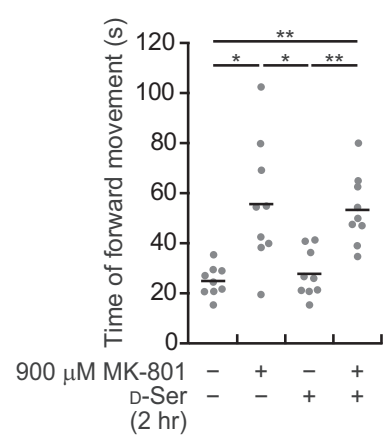

E

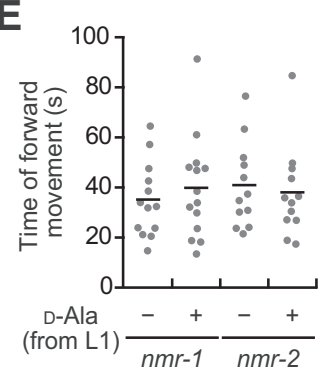

B

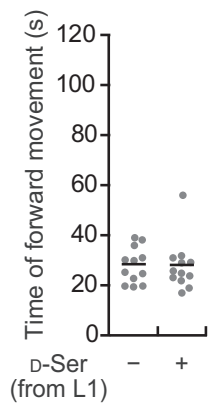

$\mathbf{F}$

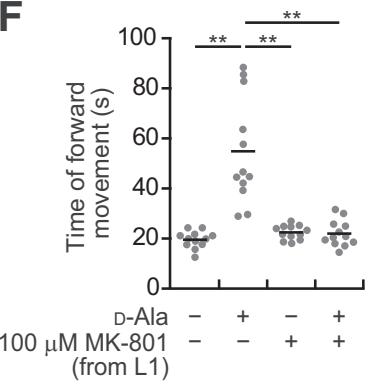

C

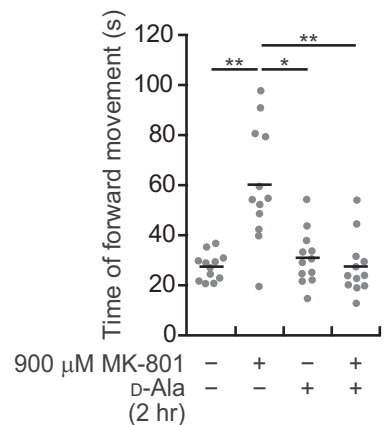

G

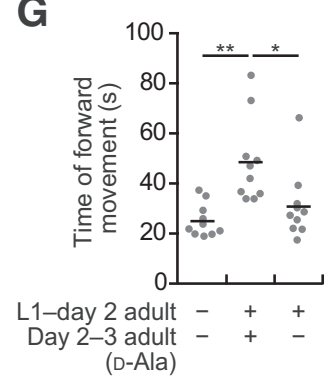

D
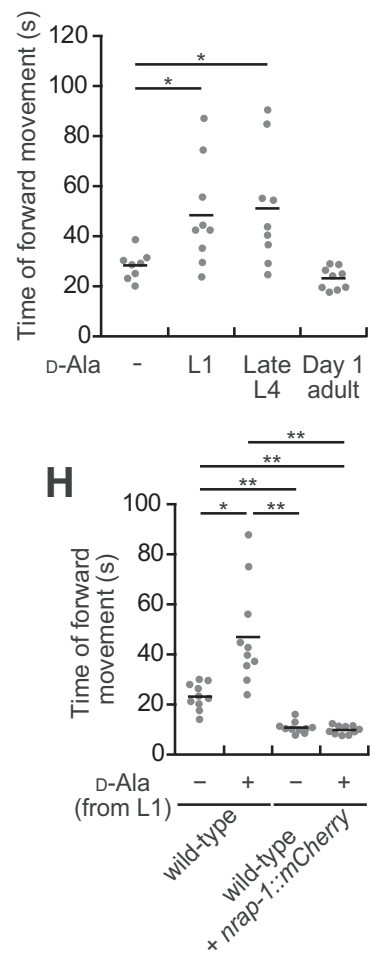

Figure 4. Effects of D-amino acid treatments on the time of forward movement immediately after food removal. Forward movement times $0-5$ min after food removal. Dots represent single worms; bars are means; ${ }^{*} p<0.05$ and ${ }^{* *} p<0.01$ indicate significant differences. $\boldsymbol{A}$, Effects of short-term treatments with $\mathrm{D}$-Ser and high-dose MK-801 on the times of forward movement of wilD-type worms. Day 2 adults were cultured for $2 \mathrm{~h}$ on seed agar plates containing $900 \mu \mathrm{m}$ MK-801 and/or $10 \mathrm{~mm}$ D-Ser. $\boldsymbol{B}$, Effect of long-term treatment with D-Ser on the time of forward movement of wilD-type worms. L1 larvae were cultured to day 2 adults on seed agar plates containing $10 \mathrm{~mm}$ D-Ser. C, Effects of short-term treatments with D-Ala and high-dose MK-801 on the times of forward movement of wild-type worms. Day 2 adults were cultured for $2 \mathrm{~h}$ on seed agar plates containing $900 \mu \mathrm{m}$ MK-801 and/or $10 \mathrm{~mm}$ D-Ala. D, Effects of long-term treatments with D-Ala on the times of forward movement of wilD-type worms. The worms of L1 larvae, late L4 larvae, or day 1 adults were cultured to day 2 adults on seed agar plates containing 10 mM D-Ala. $\boldsymbol{E}$, Effects of long-term treatments with D-Ala on the times of forward movement of nmr-1(ak4) and nmr-2(tm3785) worms. L1 larvae were cultured to day 2 adults on seed agar plates containing 10 mm D-Ala. $\boldsymbol{F}$, Effects of long-term treatments with D-Ala and low-dose MK-801 on the times of forward movement of wild-type worms. L1 larvae were cultured to day 2 adults on seed agar plates containing $10 \mathrm{~mm}$ D-Ala and/or $100 \mu \mathrm{m}$ MK-801. G, Times of forward movements of the day 3 adults. Wilo-type day 2 adults were treated with 10 mm D-Ala beginning at the L1 larva stage and cultured for $1 \mathrm{~d}$ on seed drug-free agar plates. $\boldsymbol{H}$, Effects of long-term treatments with D-Ala on the times of forward movement of transgenic wilo-type worms expressing the nrap-1::mCherry transgene. L1 larvae were cultured to day 2 adults on seed agar plates containing $10 \mathrm{~mm}$ D-Ala. For details, see Materials and Methods, Experimental design and statistical analysis.

was abolished by cotreatment with the relatively low dose $(100$ $\mu \mathrm{M})$ of MK-801 (Games-Howell test, $t_{(21.42)}=2.82, p=0.046$ between the NMDA-treated and cotreated group; Fig. $3 F$ ) as well as after the L1 larva had been transferred to plates without NMDA for $1 \mathrm{~d}$ (Tukey-Kramer test, $t=5.13, p<0.001$; Fig. $3 G$ ). All worms tested in Figure $3 D-G$ moved coordinately and exhibited no obvious change in the backward movement time (data not shown). No neuronal death was observed in $n m r-1$ expressing neurons labeled by $\mathrm{P} n m r-1: \mathrm{GFP}$, in which GFP is expressed under the control of the $n m r-1$ promoter (Brockie et al., 2001a), with long-term NMDA treatment (Fig. 3H). Therefore, the increased time of forward movement of wild-type worms treated with NMDA long-term is reversible and may reflect receptor desensitization, as prolonged exposure to NMDA produces a time-dependent decrease in NMDA-dependent currents (Brockie et al., 2001b; Traynelis et al., 2010).

C. elegans NRAP-1 is a presynaptically released NMDA receptor auxiliary protein that has been reported to act as a negative regulator of receptor desensitization (Lei et al., 2017). Overexpression of NRAP-1 enhances NMDA-induced current and decreases forward movement time compared with those in wild-type worms. To address whether the NMDA-induced increased forward movement time involves NMDA receptor desensitization, we generated transgenic wild-type worms that expressed a fusion protein of NRAP-1 and mCherry under the control of the nrap-1 promoter (nrap-1::mCherry). Long-term treatment of the transgenic L1 larva with $5 \mathrm{~mm}$ NMDA did not affect the time spent moving forward (Games-Howell test, $t_{(17.41)}=0.14, p=0.999$; Fig. 3I). All worms tested in Figure $3 I$ moved coordinately and exhibited no obvious change in the backward movement time (data not shown). The results support the hypothesis that the increased forward movement time of wild-type worms treated with NMDA long-term reflects receptor desensitization.

\section{D-Amino acids influence forward movement immediately after food removal}

We next examined whether D-Ser and D-Ala affect the time of forward movement immediately after food removal. Short-term treatment with $10 \mathrm{~mm} \mathrm{D}$-Ser did not affect the time of forward movement of wild-type worms that were untreated or treated with high-dose MK-801 (Games-Howell test: $t_{(11.91)}=0.71, p=$ 0.893 between the untreated and $\mathrm{D}$-Ser-treated group; $t_{(19.64)}=$ $0.56, p=0.943$ between the MK-801-treated and cotreated group; Fig. 4A). Similarly, long-term treatment of wild-type L1 larva with $10 \mathrm{~mm}$ D-Ser did not affect the time spent moving forward (Student's unpaired $t$ test, $t_{(22)}=0.08, p=0.934$; Fig. $4 B$ ). By contrast, $10 \mathrm{~mm}$ D-Ala abolished the increase in the time of forward 
movement produced by short-term treatment with high-dose MK-801 (Games-Howell test, $t_{(16.24)}=4.39, p=0.002$ between the MK-801-treated and cotreated group; Fig. 4C). Long-term treatments with $10 \mathrm{~mm} \mathrm{D}$-Ala, beginning at larval stages L1 or late L4, significantly increased the time of forward movement of wild-type worms (Games-Howell test: L1, $t_{(2.55)}=7.87, p=0.041$; late L4, $t_{(2.90)}=7.87, p=0.018$; Fig. $\left.4 D\right)$ but not $n m r-1(a k 4)$ or $n m r-2(t m 3785)$ single mutants (Student's unpaired $t$ test: $n m r-1$ (ak4), $t_{(25)}=0.68, p=0.506 ; n m r-2\left(\right.$ tm3785), $t_{(22)}=0.40, p=0.690$; Fig. $4 E$ ). Furthermore, this increase in forward movement in wild-type worms with long-term D-Ala treatment was abolished by cotreatment with low-dose MK-801 (Games-Howell test, $t_{(12.33)}=5.17, p=0.001$ between the D-Ala-treated and cotreated group; Fig. $4 F$ ) and was reversible (Tukey-Kramer test, $t=2.97$, $p=0.016$; Fig. $4 G$ ). Long-term D-Ala treatment of transgenic wild-type worms carrying the nrap-1::mCherry transgene did not affect the time spent moving forward (Games-Howell test, $t_{(16.41)}=1.05, p=0.724$; Fig. $\left.4 H\right)$. All worms tested in Figure $4 A-H$ moved coordinately and exhibited no obvious change in the backward movement time (data not shown). The nmr-1expressing neurons labeled by $\mathrm{P} n m r-1:: \mathrm{GFP}$ with long-term DAla treatment were alive (Fig. $3 H$ ). Thus, D-Ala and NMDA similarly influence the forward movement of wild-type worms, and D-Ala therefore appears to act as an agonist of C. elegans NMDA receptors.

\section{The increased time of forward movement of daao-1 mutants immediately after food removal involves reduced NMDA receptor activity, SERR-1, and D-Ser}

We next sought to determine the molecular mechanism underlying the increase in time spent moving forward immediately after food removal in daao-1(tm3673) mutants (Fig. 1C). Short-term treatment with high-dose MK-801 did not further increase the time of forward movement of daao-1(tm3673) mutants (Student's unpaired $t$ test, $t_{(12)}=0.09, p=0.927$; Fig. $5 A$ ). Furthermore, the times of forward movement were similar among daao-1(tm3673), $n m r-1(a k 4)$, and daao-1(tm3673);nmr-1(ak4) double mutants as well as $n m r-2(t m 3785)$ and daao-1(tm3673);nmr-2(tm3785) double mutants (Games-Howell test, $p \geq 0.970$ among each mutant; Fig. $5 B$ ). Long-term treatments with $5 \mathrm{~mm}$ NMDA or $10 \mathrm{~mm} \mathrm{D-}$ Ala or D-Ser beginning at the L1 larva stage did not further increase the time of forward movement of daao-1(tm3673) mutants (Dunnett's test: NMDA, $t=0.44, p=0.947$; D-Ser, $t=0.19$, $p=0.995$; D-Ala, $t=0.10, p=0.999$; Fig. $5 C$ ), but long-term treatment with low-dose MK-801 (Games-Howell test, $t_{(12.68)}=4.83$, $p=0.002$; Fig. $5 D)$ and expression of the nrap-1::mCherry transgene (Games-Howell test, $t_{(7.30)}=6.57, p=0.001$; Fig. $5 E$ ) abolished the increase. Similarly, long-term treatments with NMDA, D-Ala, or D-Ser beginning at the L1 larva stage did not influence the time of forward movement of serr-1(tm1988) mutants (Dunnett's test: NMDA, $t=0.12, p=0.999$; D-Ser, $t=0.37, p=$ 0.968 ; D-Ala, $t=0.50, p=0.928$; Fig. $5 F)$. However, the forward movement time of daao-1(tm3673);serr-1(tm1988) double mutants was increased to a level similar to that of daao-1(tm3673) single mutants with long-term treatment of $10 \mathrm{~mm}$ D-Ser (Games-Howell test, $t_{(16.24)}=3.89, p=0.006$ between the untreated and D-Ser-treated daao-1(tm3673);serr-1(tm1988); Fig. 5G). All worms tested in Figure $5 A-G$ moved coordinately and exhibited no obvious change in the backward movement time (data not shown). The nmr-1expressing neurons labeled by $\mathrm{P} n m r-1::$ GFP exhibited no obvious change in the daao-1(tm3673) and serr-1(tm1988) single mutant background on food and $60 \mathrm{~min}$ after food removal (Fig. $5 \mathrm{H}$ ). Altogether, these results suggest that the increased time of forward movement observed in daao-1(tm3673) mutants is because of NMDA receptor desensitization during food deprivation that requires D-Ser synthesized via SERR-1.

\section{Long-range dispersal behavior involves NMDA receptor} function that is regulated cooperatively by D-Ser and D-Ala We hypothesized that the increased observed time spent moving forward was a result of the worms employing long-range dispersal behavior immediately after food removal. We thus compared the duration of forward movement immediately $(0-5 \mathrm{~min})$ after food removal with that after prolonged $(60-65 \mathrm{~min})$ food deprivation. Although nmr-1(ak4), nmr-2(tm3785), nmr-1(ak4);nmr-2 (tm3785) mutants, and transgenic nrap-1::mCherry lines spent significantly more time moving forward at 60-65 min than at 05 min after food removal (Welch's unpaired $t$ test: $n m r-1(a k 4)$, $t_{(18.76)}=2.63, p=0.016 ; n m r-2(\operatorname{tm} 3785), t_{(20.78)}=2.95, p=0.008$; $n m r-1(a k 4) ; n m r-2(t m 3785), \quad t_{(12.08)}=3.07, \quad p=0.010 ; \quad$ nrap-1:: $m$ Cherry, $t_{(21.13)}=4.49, p<0.001$; Fig. $\left.6 A\right)$, the magnitude of this increase was less than that in wild-type worms. This suggests that NMDA receptor function contributes to long-range dispersal behavior. Indeed, long-term treatment of L1 larva with lowdose MK-801 partially suppressed the increase in time spent moving forward after $60-65 \mathrm{~min}$ of food deprivation, indicative of long-range dispersal behavior, in wild-type worms (GamesHowell, $t_{(20.98)}=3.44, p=0.012$ between the untreated and MK801-treated wild-type worms 60-65 min after food removal; Fig. $6 B)$ but not in $n m r-1(a k 4) ; n m r-2(t m 3785)$ mutants (Student's unpaired $t$ test, $t_{(17)}=0.75, p=0.465$; Fig. $\left.6 B\right)$. Moreover, this longrange dispersal behavior was rescued in serr-1(tm1988) mutant L1 larva treated long-term with $10 \mathrm{~mm}$ D-Ser but not $10 \mathrm{~mm}$ D-Ala or L-Ser (Games-Howell test: D-Ser, $t_{(11.25)}=4.33, p=0.005$; D-Ala, $t_{(23.23)}=0.58, p=0.937$; L-Ser, $t_{(15.78)}=0.20, p=0.997$; Fig. $\left.6 C\right)$. All worms tested in Figure $6 A-C$ moved coordinately and exhibited no obvious change in the backward movement time (data not shown). Thus, SERR-1-dependent synthesis of D-Ser is also required for the long-range dispersal behavior after prolonged food deprivation. Collectively, the results demonstrate that prolonged activation of NMDA receptors as a result of increased levels of D-amino acids leads to receptor desensitization and a change in the foraging strategy of $C$. elegans.

\section{Discussion}

Synthesis and degradation of D-Ser and D-Ala in C. elegans Although a previous study reported that D-Ser in C. elegans is biosynthesized in a serr-1-independent manner (Katane et al., 2016), we found that its synthesis within $10 \mathrm{~min}$ of food removal is dependent on serr-1 (Fig. 2A). Furthermore, this synthesis may occur as early as $1 \mathrm{~min}$ after food removal, as behavioral analyses showed that the increase in time spent moving forward by daao1(tm3673) mutants, in which D-Ser accumulates, within 5 min of food removal was inhibited completely by deletion of SERR-1 expression but restored by long-term treatment with D-Ser (Fig. $5 G$ ). In support of these results, serr-1 expression during food deprivation was highest $1 \mathrm{~min}$ after food removal, consistent with when D-Ser synthesis by SERR-1 is initiated (Fig. 2E). Therefore, it is likely that feedback control of serr-1 expression is required to maintain a sufficiently elevated level of D-Ser. In PAT-Seq analysis, serr-1 is expressed in the intestine and hypodermis but not in nmr-1-expressing neurons (Blazie et al., 2017). Interestingly, Drosophila SerR is expressed in the intestine and neurons, and intestinal but not neuronal expression of SerR regulates sleep through the NMDA receptor (Dai et al., 2019). These findings 
A

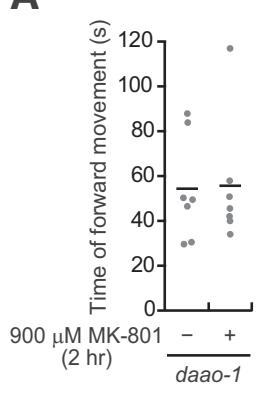

B

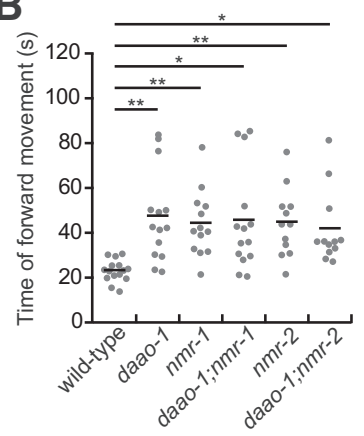

E

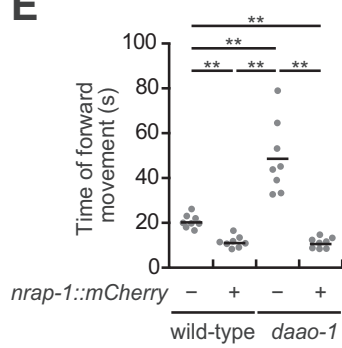

F

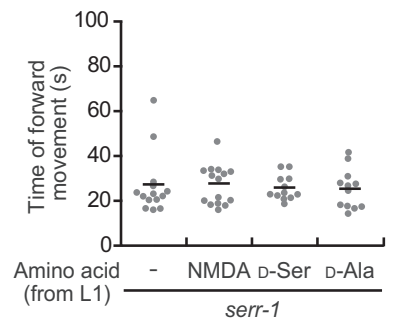

C

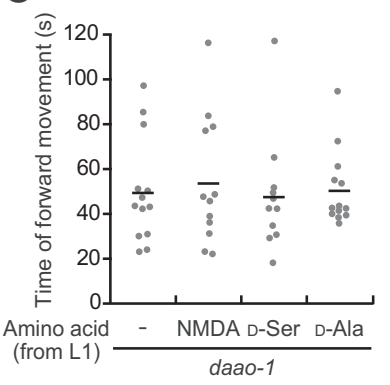

D

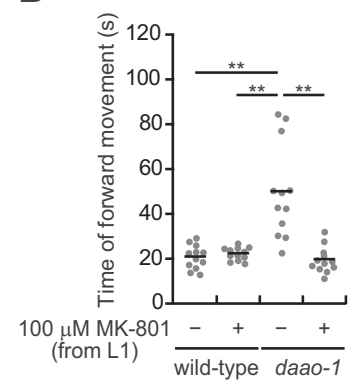

G

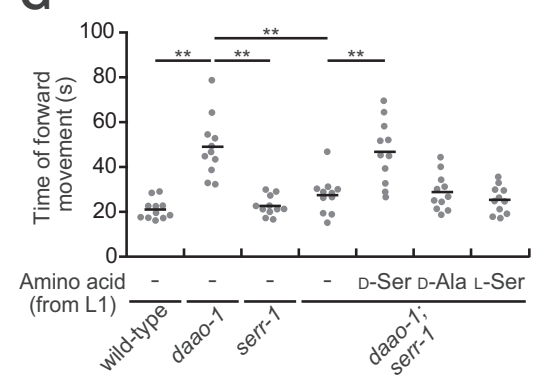

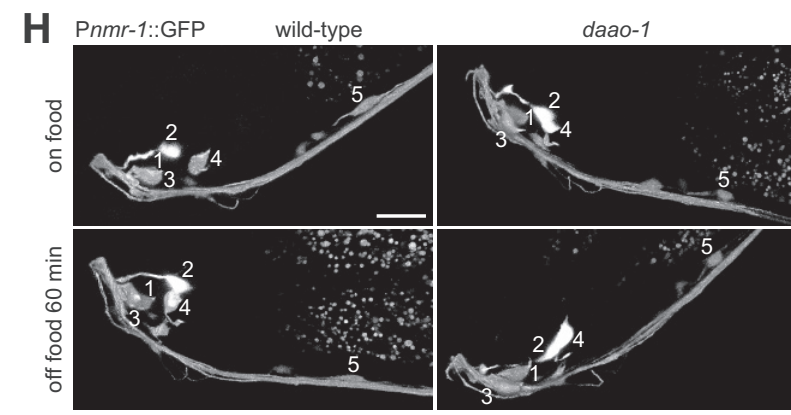

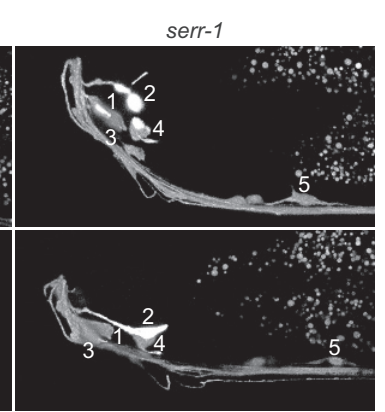

Figure 5. Time of forward movement of daao-1(tm3673) mutants immediately after food removal increases via NMDA receptor desensitization that requires D-Ser synthesized through SERR-1. $A-G$, Forward movement times $0-5$ min after food removal. Dots represent single worms; bars are means; ${ }^{*} p<0.05$ and ${ }^{* *} p<0.01$ indicate significant differences. $\boldsymbol{A}$, Effect of short-term treatment with high-dose MK-801 on the time of forward movement of daao-1(tm3673) worms. Day 2 adults were cultured for $2 \mathrm{~h}$ on seed agar plates containing $900 \mu \mathrm{m}$ MK-801. $\boldsymbol{B}$, Times of forward movement of daao-1 (tm3673);nmr-1(ak4) and daao-1(tm3673);nmr-2(tm3785) worms. C, Effects of long-term treatments with NMDA, D-Ser, and D-Ala on the times of forward movement of daao-1 (tm3673) worms. L1 larvae were cultured to day 2 adults on seed agar plates containing $5 \mathrm{~mm} \mathrm{NMDA,} 10 \mathrm{~mm}$ D-Ser, or $10 \mathrm{~mm}$ D-Ala. D, Effects of long-term treatment with low-dose MK-801 on the time of forward movement of daao-1(tm3673) worms. L1 larvae were cultured to day 2 adults on seed agar plates containing $100 \mu \mathrm{M}$ MK-801. $\boldsymbol{E}$, Times of forward movement of transgenic daao-1 (tm3673) mutants expressing the nrap-1::mCherry transgene. $\boldsymbol{F}$, Effects of long-term treatments with NMDA, D-Ser, and D-Ala on the time of forward movement of serr-1 (tm1988) worms. L1 larvae were cultured to day 2 adults on seed agar plates containing 5 mM NMDA, 10 mM D-Ser, or 10 mM D-Ala. G, Effects of long-term treatments with D-Ser, D-Ala, and L-Ser on the time of forward movement of daao-1(tm3673);serr-1(tm1988) worms. L1 larvae were cultured to day 2 adults on seed agar plates containing $10 \mathrm{~mm} \mathrm{D-Ser,} \mathrm{D-Ala,} \mathrm{or} \mathrm{L-Ser.} \boldsymbol{H}$, Confocal stack images of transgenic worms expressing the Pnmr-1::GFP transgene in the daao-1(tm3673) and serr-1(tm 1988) single mutant background on food and 60 min after food removal. At least one of the four pairs of interneurons (1, AVA; 2, AVD; 3, AVE; 4, RIM) and an interneuron (5, AVG) in the left-side head region are shown. Scale bar: $20 \mu \mathrm{m}$. For details, see Materials and Methods, Experimental design and statistical analysis.

suggest that SERR-1 expressed in the intestine is involved in the synthesis of D-Ser during food deprivation. However, the low SerR activity of recombinant SERR-1 (Katane et al., 2016; Uda et al., 2016) raises the question as to whether D-Ser is synthesized by only SERR-1. The levels of D-Ser were similar in well-fed serr-1 (tm1988) mutants and wild-type worms, but did not change in the mutants during food deprivation (Fig. $2 A$ ), indicating that levels when food is available are derived via synthesis through an enzyme other than SERR-1. Indeed, the glycine cleavage system has been implicated in D-Ser synthesis in the mammalian brain (Iwama et al., 1997).

The origin of D-Ala in fed and food-deprived C. elegans remains largely unknown. However, our results suggest that it is synthesized as early as $1 \mathrm{~min}$ after food removal (Fig. 2B). C. elegans does not harbor alanine racemase (EC 5.1.1.1), an enzyme that converts L-Ala to D-Ala. Interestingly, E. coli harbors a cystathionine $\beta$-lyase (EC 4.4.1.8), MetC, which exhibits relatively high alanine racemase activity (Soo et al., 2016; Miyamoto et al., 2018). We are currently investigating whether homologous genes in C. elegans (cbl-1, cth-1, and cth-2) are responsible for the in vivo metabolism of D-Ala.

Although it was previously unclear whether DAAO-1 regulates endogenous D-Ser and D-Ala levels in C. elegans, our results indicate that DAAO- 1 degrades these D-amino acids after food deprivation (Fig. $2 A, B$ ). In transgenic worms that expressed the daao-1::mCherry construct, DAAO-1::mCherry localization was observed in the intestine during food deprivation (Fig. 1F). Thus, DAAO-1 is presumed to be responsible for the degradation of these D-amino acids in the intestine. Previously, we reported that the transcriptional localization of DAAO-1 is observed exclusively in intestinal cells (Saitoh et al., 2012). However, in the present study, the localization of DAAO-1::mCherry was observed in the coelomocytes as well (Fig. 1F). Because coelomocytes are scavenger cells that continuously and nonspecifically endocytose 
A

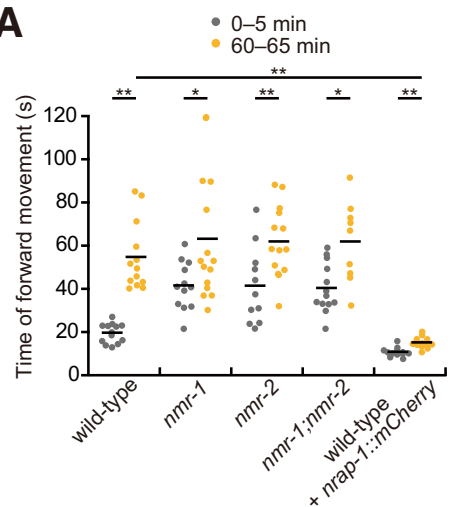

B

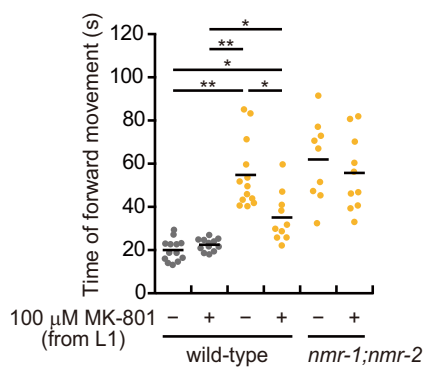

C

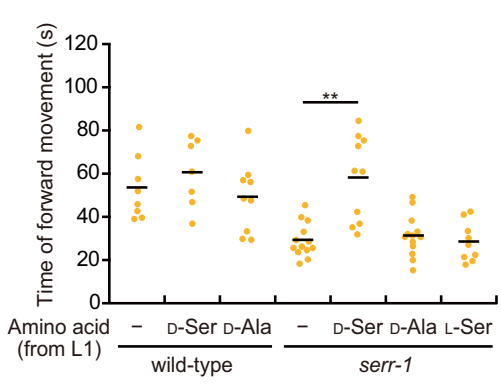

Figure 6. Long-range dispersal behavior after prolonged food deprivation requires NMDA receptor desensitization regulated by D-Ser and D-Ala. Forward movement times $0-5$ min or 60-65 min after food removal. Dots represent single worms; bars are means; ${ }^{*} p<0.05$ and ${ }^{* *} p<0.01$ indicate significant differences. $\boldsymbol{A}$, Times of forward movement of $n m r-1(a k 4)$, $n m r-2$ (tm3785), nmr-1(ak4);nmr-2(tm3785), and transgenic wilo-type worms carrying the nrap-1::mCherry transgene 60-65 min after food removal. $\boldsymbol{B}$, Effects of long-term treatments with low-dose MK-801 on the times of forward movement of wild-type and nmr-1(ak4);nmr-2(tm3785) worms 60-65 min after food removal. L1 larvae were cultured to day 2 adults on seed agar plates containing $100 \mu \mathrm{m}$ MK-801 before being transferred to transition plates containing MK-801 for $60 \mathrm{~min}$. C, Effects of long-term treatments with D-Ser, D-Ala, and L-Ser on the times of forward movement of wild-type and serr-1(tm1988) worms 60-65 min after food removal. L1 larvae were cultured to day 2 adults on seed agar plates containing 10 mm D-Ser, D-Ala, or L-Ser before being transferred to transition plates containing D-Ser, D-Ala, or L-Ser for $60 \mathrm{~min}$. For details, see Materials and Methods, Experimental design and statistical analysis.

fluid from the body cavity (Fares and Grant, 2002), these results implied that DAAO- 1 is transferred into the body fluid from the intestinal cells that produce it and is then taken up by coelomocytes, in which it is degraded.

\section{Applications of NMDA and MK-801 in C. elegans foraging behavior}

MK-801 is a noncompetitive open-channel blocker and appears to inhibit the activity of the C. elegans NMDA receptor in a similar manner because its binding sites are conserved in NMR-1 (Brockie et al., 2001b). In mice, the ability of MK-801 to potentiate EtOH-induced ataxia and sedation/hypnosis depends on the activity of AMPA receptors that are closely involved with NMDA receptors (Palachick et al., 2008). However, the localization and function of AMPA receptors in C. elegans are not dependent on NMDA receptors (Brockie et al., 2001b), and shortterm treatments with $900 \mu \mathrm{M}$ MK-801 did not further increase the time spent moving forward in NMDA receptor-null mutants (Fig. $3 B)$, suggesting that MK-801 acted on NMDA receptors, leading to its behavioral effect. In wild-type worms, short-term treatment with $900 \mu \mathrm{M}$ MK-801 may inhibit the activity of a population of NMDA receptors in an endogenous agonist-bound state, result-

ing in an increase in the time spent moving forward (Fig. 3B,C). By contrast, the long-term treatment with $100 \mu \mathrm{M}$ MK-801 may inhibit the activity of a smaller population of endogenous agonist-bound NMDA receptors at a level not sufficient to increase the time spent moving forward (Fig. 3F). When both NMDA and $900 \mu \mathrm{M}$ MK-801 were coapplied in the short-term, the behavioral effect of $900 \mu \mathrm{M}$ MK-801 was abolished completely (Fig. $3 C$ ). This dominance of coapplied NMDA may be because the $C$. elegans NMDA receptor blockade by MK-801 is partial and reversible in patch-clamp recording (Brockie et al., 2001b; Lei et al., 2017).

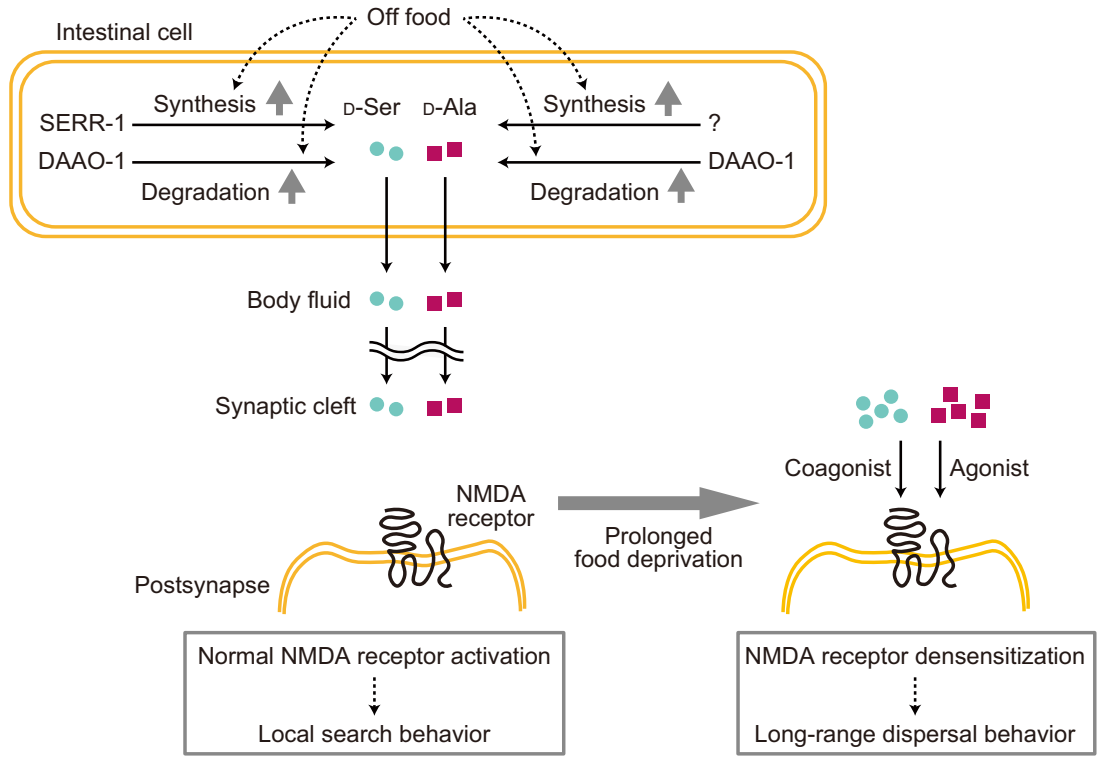

Figure 7. Schematic illustration of the possible mechanism of foraging behavior during food deprivation. Food deprivation generates a high frequency of spontaneous reversal, resulting in the local search behavior that requires normal NMDA receptor activation. Simultaneously, in the intestine, D-Ser is synthesized via SERR-1 and D-Ala is synthesized via an unidentified pathway. The levels of these D-amino acids are also regulated by DAA0-1. With prolonged food deprivation, the elevated levels of synaptic D-Ser and D-Ala through intestinal release and body fluid transport cooperatively activate NMDA receptors as a coagonist and an agonist, respectively, and induce NMDA receptor desensitization resulting in a lower frequency of spontaneous reversals and thus long-range dispersal behavior.

This study indicated that the increased time spent moving forward in wild-type worms treated with NMDA long-term reflects NMDA receptor desensitization (Fig. 3I). Short-term NMDA treatment may have also led to a small population of desensitized NMDA receptors at a level not sufficient to cause the behavioral effect (Fig. 3C) because receptor desensitization is in the order of minutes (Brockie et al., 2001b). When both NMDA and $100 \mu \mathrm{M}$ MK-801 were coapplied long-term, MK-801 may inhibit the receptor desensitizationinducing activity in a large population of NMDA receptors in the NMDA-bound state, resulting in a normal time spent moving forward (Fig. $3 F$ ). 


\section{Elevated levels of D-Ser and D-Ala promote long-range dispersal behavior after prolonged food deprivation}

Application of D-Ser for long-term did not increase the time of forward movement of wild-type worms immediately after food removal (Fig. 4B), suggesting that the NMDA receptors saturated only with $\mathrm{D}$-Ser are not desensitized to a level that affects the behavior. This may be because D-Ser is a coagonist (not an agonist) of the NMDA receptor. Indeed, so far, there is no report showing the receptor desensitization in response to increasing concentrations of D-Ser alone. We also showed that D-Ala acts as an NMDA receptor agonist in C. elegans, as it resulted in a behavior similar to that by NMDA (Figs. $3 C-G, I, 4 C-H$ ). Thus, the action of D-Ala on NMDA receptors in C. elegans is different from that in mammals. Moreover, D-Ser synthesized via SERR-1 was required for the increased forward movement time in daao1(tm3673) mutants immediately after food removal (Fig. 5G). This behavior may result from the NMDA receptor desensitization following excessive activation by D-Ala acting as an agonist on the receptors with a D-Ser bound to the receptor. On the other hand, we surmise that after prolonged food deprivation, NMDA receptor desensitization following activation by D-Ala and D-Ser synthesized via SERR-1 contributes to long-range dispersal behavior (Fig. 6). Although whole-body D-Ala levels in wildtype worms did not increase after food deprivation (Fig. 2B), it is possible that levels around or within the command interneurons increase to cooperatively desensitize NMDA receptors if the worms are capable of D-Ala biosynthesis. Interestingly, serr1(tm1988) mutants exhibited normal foraging behaviors immediately after food removal (Fig. $1 L-N$ ), suggesting that D-Ser synthesized via SERR-1 is not essential for NMDA receptor activation regulating local search behavior. Namely, the receptor-binding of D-Ser may be required only for the receptordesensitization. This seems reasonable for the regulation of long-range dispersal behavior involved in receptor-desensitization. Electrophysiological studies will help to elucidate the effects of D-Ser and D-Ala on NMDA receptors in C. elegans.

Long-range dispersal behavior after prolonged food deprivation also involves mechanisms other than the regulation of NMDA receptor activity, because wild-type worms treated longterm with a low-dose of MK-801 as well as the NMDA receptornull mutants and transgenic nrap-1::mCherry lines exhibited a small increase in the time spent moving forward during prolonged food deprivation (Fig. 6A,B). Remarkably, this increase in wild-type worms was completely dependent on D-Ser synthesized via SERR-1 (Fig. 6C), indicating that the newly synthesized D-Ser contributes to mechanisms other than those involving NMDA receptor activity, such as serotonergic activation (Wakabayashi et al., 2005). For example, D-Ser can bind to serotonin 5-HT3 receptors on human platelets (Fatima Shad, 2006).

Collectively, the results of the present study suggest that D-Ser and D-Ala are endogenous regulators that control adaptive foraging behavior in C. elegans via the NMDA receptor (Fig. 7). In these worms, food deprivation activates NMDA receptors to generate the local search behavior and induce the intestinal synthesis of D-Ser via SERR-1 and D-Ala through an unidentified pathway. With prolonged food deprivation, the continued activation of NMDA receptors via elevated levels of synaptic D-Ser and D-Ala through intestinal release and body fluid transport, acting as a coagonist and agonist, respectively, results in receptor desensitization that leads to long-range dispersal behavior. Thus, SERR-1 and DAAO-1 play important roles in the regulation of D-Ser and D-Ala levels during food deprivation and subsequent foraging strategies.

\section{References}

Bendikov I, Nadri C, Amar S, Panizzutti R, De Miranda J, Wolosker H, Agam G (2007) A CSF and postmortem brain study of D-serine metabolic parameters in schizophrenia. Schizophr Res 90:41-51.

Blazie SM, Geissel HC, Wilky H, Joshi R, Newbern J, Mangone M (2017) Alternative polyadenylation directs tissue-specific miRNA targeting in Caenorhabditis elegans somatic tissues. Genetics 206:757-774.

Brenner S (1974) The genetics of Caenorhabditis elegans. Genetics 77:71-94.

Brockie PJ, Madsen DM, Zheng Y, Mellem J, Maricq AV (2001a) Differential expression of glutamate receptor subunits in the nervous system of Caenorhabditis elegans and their regulation by the homeodomain protein UNC-42. J Neurosci 21:1510-1522.

Brockie PJ, Mellem JE, Hills T, Madsen DM, Maricq AV (2001b) The C. elegans glutamate receptor subunit NMR-1 is required for slow NMDAactivated currents that regulate reversal frequency during locomotion. Neuron 31:617-630.

Chalfie M, Sulston J (1981) Developmental genetics of the mechanosensory neurons of Caenorhabditis elegans. Dev Biol 82:358-370.

Chalfie M, Sulston JE, White JG, Southgate E, Thomson JN, Brenner S (1985) The neural circuit for touch sensitivity in Caenorhabditis elegans. J Neurosci 5:956-964.

Dai X, Zhou E, Yang W, Zhang X, Zhang W, Rao Y (2019) D-Serine made by serine racemase in Drosophila intestine plays a physiological role in sleep. Nat Commun 10:1986.

Fares H, Grant B (2002) Deciphering endocytosis in Caenorhabditis elegans. Traffic 3:11-19.

Fatima Shad K (2006) Effect of D-serine on the serotonin receptors of human platelets. Exp Brain Res 173:353-356.

Gray JM, Hill JJ, Bargmann CI (2005) A circuit for navigation in Caenorhabditis elegans. Proc Natl Acad Sci USA 102:3184-3191.

Hashimoto A, Nishikawa T, Oka T, Takahashi K (1993) Endogenous D-serine in rat brain: $N$-methyl-D-aspartate receptor-related distribution and aging. J Neurochem 60:783-786.

Henneberger C, Papouin T, Oliet SHR, Rusakov DA (2010) Long-term potentiation depends on release of D-serine from astrocytes. Nature 463:232-236.

Hills T, Brockie PJ, Maricq AV (2004) Dopamine and glutamate control area-restricted search behavior in Caenorhabditis elegans. J Neurosci 24:1217-1225.

Inoue R, Hashimoto K, Harai T, Mori H (2008) NMDA- and beta-amyloid142 -induced neurotoxicity is attenuated in serine racemase knock-out mice. J Neurosci 28:14486-14491.

Iwama H, Takahashi K, Kure S, Hayashi F, Narisawa K, Tada K, Mizoguchi M, Takashima S, Tomita U, Nishikawa T (1997) Depletion of cerebral Dserine in non-ketotic hyperglycinemia: possible involvement of glycine cleavage system in control of endogenous D-serine. Biochem Biophys Res Commun 231:793-796.

Kano T, Brockie PJ, Sassa T, Fujimoto H, Kawahara Y, Iino Y, Mellem JE, Madsen DM, Hosono R, Maricq AV (2008) Memory in Caenorhabditis elegans is mediated by NMDA-type ionotropic glutamate receptors. Curr Biol 18:1010-1015.

Kaplan JM, Horvitz HR (1993) A dual mechanosensory and chemosensory neuron in C. elegans. Proc Natl Acad Sci USA 90:2227-2231.

Katane M, Saitoh Y, Seida Y, Sekine M, Furuchi T, Homma H (2010) Comparative characterization of three $\mathrm{D}$-aspartate oxidases and one $\mathrm{D}$ amino acid oxidase from Caenorhabditis elegans. Chem Biodivers 7:1424-1434.

Katane M, Saitoh Y, Uchiyama K, Nakayama K, Saitoh Y, Miyamoto T, Sekine M, Uda K, Homma H (2016) Characterization of a homologue of mammalian serine racemase from Caenorhabditis elegans: the enzyme is not critical for the metabolism of serine in vivo. Genes Cells 21:966-977.

Kleckner NW, Dingledine R (1988) Requirement for glycine in activation of NMDA-receptors expressed in Xenopus oocytes. Science 241:835-837.

Krebs HA (1935) Metabolism of amino acids: deamination of amino acids. Biochem J 29:1620-1644.

Kuryatov A, Laube B, Betz H, Kuhse J (1994) Mutational analysis of the glycine-binding site of the NMDA receptor: structural similarity with bacterial amino acid-binding proteins. Neuron 12:1291-1300.

Lei N, Mellem JE, Brockie PJ, Madsen DM, Maricq AV (2017) NRAP-1 is a presynaptically released NMDA receptor auxiliary protein that modifies synaptic strength. Neuron 96:1303-1316. 
Lewis JA, Fleming JT (1995) Basic culture methods. Methods Cell Biol 48:329.

Ly K, Reid SJ, Snell RG (2015) Rapid RNA analysis of individual Caenorhabditis elegans. MethodsX 2:59-63.

Mello CC, Kramer JM, Stinchcomb D, Ambros V (1991) Efficient gene transfer in C. elegans: extrachromosomal maintenance and integration of transforming sequence. EMBO J 10:3959-3970.

Miedel MT, Graf NJ, Stephen KE, Long OS, Pak SC, Perlmutter DH, Silverman GA, Luke CJ (2012) A pro-cathepsin L mutant is a luminal substrate for endoplasmic-reticulum-associated degradation in C. elegans. PLoS One 7:e40145.

Morales-Martínez A, Dobrzynska A, Askjaer P (2015) Inner nuclear membrane protein LEM-2 is required for correct nuclear separation and morphology in C. elegans. J Cell Sci 128:1090-1096.

Miyamoto T, Katane M, Saitoh Y, Sekine M, Homma H (2018) Cystathionine $\beta$-lyase is involved in D-amino acid metabolism. Biochem J 475:1397-1410.

Miyoshi Y, Hamase K, Tojo Y, Mita M, Konno R, Zaitsu K (2009) Determination of D-serine and D-alanine in the tissues and physiological fluids of mice with various $\mathrm{D}$-amino-acid oxidase activities using twodimensional high-performance liquid chromatography with fluorescence detection. J Chromatogr B Analyt Technol Biomed Life Sci 877:25062512.

Nishikawa T (2011) Analysis of free D-serine in mammals and its biological relevance. J Chromatogr B Analyt Technol Biomed Life Sci 879:31693183.

Palachick B, Chen YC, Enoch AJ, Karlsson RM, Mishina M, Holmes A (2008) Role of major NMDA or AMPA receptor subunits in MK-801 potentiation of ethanol intoxication. Alcohol Clin Exp Res 32:1479-1492.

Panatier A, Theodosis DT, Mothet JP, Touquet B, Pollegioni L, Poulain DA, Oliet SHR (2006) Glia derived D-serine controls NMDA receptor activity and synaptic memory. Cell 125:775-784.

Raizen DM, Lee RY, Avery L (1995) Interacting genes required for pharyngeal excitation by motor neuron MC in Caenorhabditis elegans. Genetics 141:1365-1382.

Rosenberg D, Artoul S, Segal AC, Kolodney G, Radzishevsky I, Dikopoltsev E, Foltyn VN, Inoue R, Mori H, Billard JM, Wolosker H (2013) Neuronal D-serine and glycine release via the Asc-1 transporter regulates NMDA receptor-dependent synaptic activity. J Neurosci 33:3533-3544.
Saitoh Y, Katane M, Kawata T, Maeda K, Sekine M, Furuchi T, Kobuna H, Sakamoto $\mathrm{T}$, Inoue $\mathrm{T}$, Arai $\mathrm{H}$, Nakagawa $\mathrm{H}$, Homma $\mathrm{H}$ (2012) Spatiotemporal localization of D-amino acid oxidase and D-aspartate oxidases during development in Caenorhabditis elegans. Mol Cell Biol 32:1967-1983

Sasabe J, Chiba T, Yamada M, Okamoto K, Nishimoto I, Matsuoka M, Aiso S (2007) D-Serine is a key determinant of glutamate toxicity in amyotrophic lateral sclerosis. EMBO J 26:4149-4159.

Soo VW, Yosaatmadja Y, Squire CJ, Patrick WM (2016) Mechanistic and evolutionary insights from the reciprocal promiscuity of two pyridoxal phosphate-dependent enzymes. J Biol Chem 291:19873-19887.

Thomas JH (1990) Genetic analysis of defecation in Caenorhabditis elegans. Genetics 124:855-872.

Traynelis SF, Wollmuth LP, McBain CJ, Menniti FS, Vance KM, Ogden KK, Hansen KB, Yuan H, Myers SJ, Dingledine R (2010) Glutamate receptor ion channels: structure, regulation, and function. Pharmacol Rev 62:405496.

Uda K, Abe K, Dehara Y, Mizobata K, Sogawa N, Akagi Y, Saigan M, Radkov AD, Moe LA (2016) Distribution and evolution of the serine/aspartate racemase family in invertebrates. Amino Acids 48:387-402.

Wakabayashi T, Kitagawa I, Shingai R (2004) Neurons regulating the duration of forward locomotion in Caenorhabditis elegans. Neurosci Res 50:103-111.

Wakabayashi T, Osada T, Shingai R (2005) Serotonin deficiency shortens the duration of forward movement in Caenorhabditis elegans. Biosci Biotechnol Biochem 69:1767-1770.

White JG, Southgate E, Thomson JN, Brenner S (1986) The structure of the nervous system of the nematode Caenorhabditis elegans. Philos Trans $\mathrm{R}$ Soc Lond B Biol Sci 314:1-340.

Wolosker H (2007) NMDA receptor regulation by D-serine: new findings and perspectives. Mol Neurobiol 36:152-164.

Wolosker H, Sheth KN, Takahashi M, Mothet JP, Brady RO Jr, Ferris CD, Snyder SH (1999) Purification of serine racemase: biosynthesis of the neuromodulator D-serine. Proc Natl Acad Sci USA 96:721-725.

Wu SZ, Bodles AM, Porter MM, Griffin WST, Basile AS, Barger SW (2004) Induction of serine racemase expression and $\mathrm{D}$-serine release from microglia by amyloid $\beta$-peptide. J Neuroinflammation 1:2. 